\title{
Biomimetic quantum dot-labeled BI6FIO murine melanoma cells as a tool to monitor early steps of lung metastasis by in vivo imaging
}

This article was published in the following Dove Press journal: International Journal of Nanomedicine

\author{
Víctor Manuel Díaz-García, ${ }^{1-4}$ \\ Simón Guerrero, ${ }^{1,2,5}$ Natalia \\ Díaz-Valdivia, ${ }^{1,2}$ Lorena Lobos- \\ González, 2,6,7 Marcelo Kogan, 2,5 \\ José Manuel Pérez-Donoso, ${ }^{3}$ \\ Andrew FG Quest ${ }^{1,2}$ \\ 'Cellular Communication Laboratory, \\ Center for Studies on Exercise, \\ Metabolism and Cancer (CEMC), Faculty of \\ Medicine, Universidad de Chile, Santiago, \\ Chile; ${ }^{2}$ Advanced Center for Chronic \\ Diseases (ACCDiS), Faculty of Medicine, \\ Universidad de Chile, Santiago, Chile; \\ ${ }^{3}$ BioNanotechnology and Microbiology \\ Laboratory, Center for Bioinformatics \\ and Integrative Biology (CBIB), Faculty of \\ life Sciences, Universidad Andres Bello, \\ Santiago, Chile; ${ }^{4}$ Facultad de Ingeniería y \\ Tecnología, Universidad San Sebastián, \\ Concepción 408087I, Chile; ${ }^{5}$ Department \\ of Pharmacological and Toxicological \\ Chemistry, Faculty of Chemical and \\ Pharmaceutical Sciences, Universidad de \\ Chile, Santiago, Chile; ${ }^{6}$ Fundación Ciencia y \\ Vida, Santiago, Chile; ${ }^{7}$ Centro de Medicina \\ Regenerativa, Facultad de Medicina, Clínica \\ Alemana, Universidad del Desarrollo, \\ Santiago, Chile
}

Background: Numerous studies have proposed the use of fluorescent semiconductor nanoparticles or quantum dots (QDs) as novel tools to label cells and tumors. However, QD applications are limited by their toxicity in biological systems and little is known about whether QDs affect the capacity of cancer cells to metastasize. Previously, we described the "biomimetic" synthesis of CdTe-QDs (QDsglutathione [GSH]) with increased biocompatibility and the potential utility in labeling cells.

Purpose: In order to determine the feasibility of using QDs-GSH as a tool for tracking tumor cells during early metastasis, we characterized here for the first time, the in vitro and in vivo effects of the incorporation of green or red biomimetic QDs-GSH into B16F10 cells, a syngeneic mouse melanoma line for metastasis assays in C57BL/6 mice.

Methods: B16F10 cells were labeled with green or red biomimetic QDs-GSH in the presence or absence of n-acetylcysteine. Then, migration, invasion and proliferation of labeled B16F10 were evaluated in vitro. Finally, the B16F10 cells labeled with red QDs-GSH were used to monitor in vivo lung metastasis at early time points (5 minutes to 24 hours) or after 21 days in C57BL/6 mice.

Results: We developed a methodology that allows obtaining QDs-GSH-labeled B16F10 cells (nearly $100 \%$ viable labeled cells), which remained viable for at least 5 days and migrated similarly to control cells. However, proliferation, invasion, and the capacity to form metastatic nodules in the lungs were severely attenuated. Fluorescence imaging revealed that distribution/accumulation of QDs-GSH-labeled B16F10 cells could be tracked following injection into C57BL/6 mice (syngeneic preclinical metastasis model) and that these cells preferentially accumulated in the perialveolar area in lungs as early as 5 minutes post-injection.

Conclusion: The methodology described here represents a useful alternative for monitoring initial events during tumor cell metastasis.

Keywords: cell tracking, biomimetic, invasion, proliferation, migration, cancer

\section{Introduction}

Cancer is a leading cause of death world-wide ${ }^{1}$ and frequently attributable to tumor cell dissemination to distant organs, a process referred to as metastasis. ${ }^{2,3}$ The evolution of metastasis in vivo is difficult to follow, mainly because only a few tumor cells that enter the blood stream successfully colonize distant organs in a clinically detectable fashion. ${ }^{3}$ Standard in vivo experimental approaches to study metastasis involve the injection of metastatic cells into the blood stream of a mouse and subsequent evaluation of distant organ invasion/colonization as metastatic foci after sacrificing the animal, generally several weeks post-inoculation of the tumor cells. Accordingly, these traditional methods are considered "black box" experiments, given that the events occurring between initial administration of cells and metastatic tumor formation in the target organ cannot be directly observed. ${ }^{4}$ 
For in vivo imaging studies, fluorescence stands out over other techniques due to its low cost, high signal as well as spatial and temporal resolution, and the possibility of simultaneously evaluating multiple labels. ${ }^{4,5}$ The main disadvantages of fluorescence-based techniques include the instability of fluorescent probes and autofluorescence of biomolecules. ${ }^{4}$ In the last decade, sensitivity, versatility and stability of fluorescence imaging applications have increased notably, thanks to the development of semiconductor nanoparticles (NPs) or quantum dots (QDs). ${ }^{4-6}$

QDs are inorganic, semiconducting, and fluorescent NPs, ranging in size from 1 to $20 \mathrm{~nm}$ approximately. ${ }^{7-9}$ The QD size determines their fluorescence emission wavelength, which may vary from $450 \mathrm{~nm}$ (blue-green) to $650 \mathrm{~nm}$ (red). ${ }^{7,8,10,11}$ QDs possess a number of spectroscopic properties that make them particularly interesting for studies in vivo: 1) elevated resistance to chemical degradation and photobleaching; 2) narrow and almost symmetric fluorescence peaks; 3) a broad light absorption spectrum that allows fine-tuning for processing of optical images and multispectral analysis of multiple objects simultaneously; $;^{7,10,11}$ and 4) a sensitivity that may be 2-3-fold higher than other organic fluorophores commonly used for detection in tissues..$^{10,11}$ The aforementioned characteristics of QDs make them a promising tool for tumor and/or cell labeling, raising the specter of being able to track essentially real-time tumor cell migration, and their accumulation in distant organs. ${ }^{12}$

A major disadvantage of using QDs in biological systems is their toxicity associated with the formation of reactive oxygen species (ROS) and the release of heavy metal ions from the QDs core (ie, $\mathrm{Cd}^{+2}$ and $\mathrm{Pb}^{+2}$, among others). ${ }^{13-16}$ These ions interact with intracellular thiolated proteins and glutathione (GSH), thereby altering the cellular redox environment ${ }^{16-19}$ and these changes likely affect the behavior of QDs-labeled metastatic cells. ${ }^{20}$ More recently, in an attempt to bypass these limitations, a new generation of cadmium-free QDs was developed, with essentially the same spectroscopic advantages but lacking detrimental effects linked to the presence of cadmium. Nonetheless, some toxic effects attributable to physical and surface properties of these QDs remain. ${ }^{21}$

In this perspective, a few years ago, we developed a protocol to synthesize, in an ecofriendly and biocompatible manner, cadmium-telluride $\mathrm{QDs}^{8}$ (Patent No US 20130284979). ${ }^{22}$ This method, referred to as "Biomimetic," permits obtaining rapidly and easily, hydrophilic QDs, stabilized with GSH on their surface (QDs-GSH), which emit fluorescence between $480 \mathrm{~nm}$ (green) and $650 \mathrm{~nm}$ (red), depending upon their size. ${ }^{8,9}$ QDs-GSH fluorescence was shown to remain stable for months when kept at room temperature. Moreover, QDs-GSH do not precipitate in buffers, cells, or bacterial culture media, in contrast to classic QDs. ${ }^{8}$ It is noteworthy that for QDs-GSH, no significant detrimental effects on human gastric cancer cell (MKN45) viability and morphology were detected after QDS-GSH internalization, even when incubated at concentrations up to $200 \mu \mathrm{g} / \mathrm{mL}^{8,9,23}$ Nevertheless, it still remained to be determined how QDs-GSH affect migration, invasion, and metastasis of cancer cells.

In the current study, we extensively characterized the effects of QDs-GSH internalization on the viability, proliferation, and invasion in vitro, as well as lung metastasis in vivo of B16F10 murine melanoma cells in syngeneic C57BL/6 mice, a preclinical melanoma model. ${ }^{24,25}$ QDs-GSH-labeled B16F10 cells were used to track the early accumulation/ distribution of these metastatic cells in C57BL/6 mice. Although QDs-GSH internalization severely reduced the ability of B16F10 cells to form visible metastatic nodules 21 days post-injection, the QDs-GSH-labeled B16F10 cells could be successfully tracked during initial colonization of distant organs including the lung.

\section{Materials and methods QDs-GSH chemical synthesis}

QDs-GSH were synthesized by protocols previously described by Pérez-Donoso et al. ${ }^{8,22}$ Citrate/ $\mathrm{NaBH}_{4} 15 \mathrm{mM}$ $(\mathrm{pH}=9.4)$ solution was used as the synthesis buffer, to which $\mathrm{CdCl}_{2} 40 \mu \mathrm{M}, \mathrm{K}_{2} \mathrm{TeO}_{3} 10 \mu \mathrm{M}$, and $\mathrm{GSH} 10 \mathrm{mM}$ were added. The synthesis solution was incubated at $90^{\circ} \mathrm{C}$ shaking vigorously for 2 (green QDs-GSH [gQDs-GSH]) or 6 hours (red QDs-GSH [rQDs-GSH]) and the synthesis reaction was stopped by cooling to $4^{\circ} \mathrm{C}$. QDs-GSH were dialyzed in Citrate/ $\mathrm{NaBH}_{4} 15 \mathrm{mM}$ at pH 9.4, during 2 hours. Afterwards, QDs-GSH were precipitated using 2 volumes of ethanol for 10 minutes at $-20^{\circ} \mathrm{C}$. Solutions were then centrifuged at $24,000 \times g$ at $4^{\circ} \mathrm{C}$ for 20 minutes. Pellets were purified and vacuum dried for 24 hours at room temperature. Finally, QDs were weighed and dissolved in nanopure water.

\section{QDs-GSH characterization}

Solutions of different QDs-GSH $(10 \mathrm{mg} / \mathrm{mL})$ were characterized by spectroscopy. Characterization was performed in 96-well plates, using a multimodal multiplate reader (fluorescence and absorbance) (Sinergy-H1M; Biotek). Size and zeta potential were determined by dynamic light scattering and laser electrophoresis Doppler, respectively (Zetasizer nano S90 light; Malvern Instruments Limited, Malvern, UK). The core size of rQDs-GSH was determined by using a JEOL/JEM 1200 EX II transmission electron microscope (Figure S1). 


\section{Cell culture and QDs-GSH incubation}

B16F10 cells (provided originally by Dr Laurence Zitvogel, Institut Gustave Roussy, Villejuif, France) were cultured for 24 hours in Roswell Park Memorial Institute medium (RPMI medium) (Gibco ${ }^{\mathrm{TM}}$; Thermo Fisher Scientific, Waltham, MA, USA) supplemented with $10 \%$ fetal bovine serum (FBS; Bioind) and antibiotics (penicillin $100 \mathrm{UI} / \mathrm{mL}$, streptomycin $100 \mu \mathrm{g} / \mathrm{mL}$ ), at $37^{\circ} \mathrm{C}$ in $5 \% \mathrm{CO}_{2}$. All procedures and the use of these cells were approved by the unit of risk prevention and biosafety, medical school, University of Chile and the institutional committee of care and use of animals (CICUA) of the University of Chile. Afterwards, QDs-GSH treatments were performed with the previously described culture medium supplemented with lipofectamine $4 \mu \mathrm{L} / \mathrm{mL}$ (Invitrogen), plus $0,50,100,200$, or $400 \mu \mathrm{g} / \mathrm{mL}$ of each QDs-GSH. N-acetylcysteine (NAC) treatments of $0,2,4,8$, or $10 \mathrm{mM}$ were performed using the same conditions, but only for rQDs-GSH.

\section{Fluorescence-activated cell sorting (FACS)}

Cell viability, labeled viable cells, mean fluorescence intensity (MFI) and ROS levels were determined by FACS flow cytometry. B16F10 cells treated with red and green QDs-GSH were washed twice with PBS, detached with trypsin-EDTA $0.5 \%$, and centrifuged at $150 \times g$ for 5 minutes. Then, cell pellets were washed with Flow Buffer (BD Biosciences, San José, CA, USA) and centrifuged as mentioned earlier. B16F10 cells treated with gQDs-GSH were incubated for 10 minutes with propidium iodide (PI) $10 \mu \mathrm{g} / \mathrm{mL}$ (Thermo Fisher Scientific) for death cell differentiation. On the other hand, B16F10 cells treated with rQDs-GSH were tested for green live cells-labeling (Calcein), with LIVE/DEAD ${ }^{\circledR}$ Viability/Cytotoxicity Kit, for mammalian cells (Thermo Fisher) dilution 1:100,000 to determine viability and with dihydrodichlorofluorescein (H2DCFDA) (Invitrogen) dilution 1:10,000 to determine ROS levels. Five thousand events were analyzed to determine size (forward scatter [FSC]) and granularity parameters (side scatter [SSC]), excluding cell debris. CellulargQDs-GSH-fluorescence, cells $\mathrm{H}_{\mathrm{H} \text { DCFDA }}$ and cells ${ }_{\text {Calcein }}$ were determined using the FITC channel (green fluorescence); rQDsGSH and PI mark were tracked in the PerCP-Cy5 channel (red fluorescence). Both emissions were obtained by laser excitation at $488 \mathrm{~nm}$. The percentage of viable labeled cells was obtained, for either red- or green-labeled cells, with the following equation:

$$
\begin{aligned}
& \% \text { of viable labeled cells } \\
& =\frac{\text { Viable QDs }- \text { GSH positible cells }}{\text { Total viable cells }}
\end{aligned}
$$

MFI was determined for viable QDs-labeled cells and control cells by FACs. Data were analyzed using the FCS
Express four plus research edition software (De Novo Software, Los Angeles, CA, USA).

\section{Confocal microscopy}

Viable cells labeled or not with QDs-GSH were evaluated using a Zeiss Microscope model LSM 700. The excitation laser was set at $488 \mathrm{~nm}$. Green and red fluorescence were tracked at 510 and $600 \mathrm{~nm}$, respectively. All optical sections obtained for co-localization studies were processed with Image J $1.47 \mathrm{v}$ software (National Institutes of Health, USA).

For invasion assays, fixed cells attached to the lower membrane surface were visualized using a Zeiss Microscope, model LSM 700. Wavelength excitation laser was set at $405 \mathrm{~nm}$. DAPI and rQDs-GSH fluorescence were tracked at 460 and $580 \mathrm{~nm}$, respectively. Images of either B16F10 or B16F10 ${ }_{\text {QDs-GSH-10NAC }}$ cells attached to the lower side of the well membrane were obtained. Both types of cells were labeled with DAPI 1:1,000 for 5 minutes. Afterwards, membranes were washed twice and fixed in DACO fluorescent solution.

\section{Fluorescence microscopy}

Membrane-attached cells after migration were visualized using a CKX41 inverted microscope and a fluorescence light source U-LH100HG (Olympus, Japan). Images were processed with ImageJ 1,47 v software (National Institutes of Health, USA).

\section{Quantification of reduced thiols}

B16F10 and MKN45 cells were cultured until they reached $\sim 90 \%$ confluence in $100 \mathrm{~mm}$ plates. Afterwards, cells were collected, washed with PBS and resuspended in $200 \mu \mathrm{L}$ sulfosalicylic acid 5\% (SSA). Samples were divided into two equivalent portions, one for total protein measurement (BCA assay) and the other for the determination of intracellular thiols. Cells were lyzed using 20 freeze/ thaw cycles and subsequently centrifuged at $32,000 \times g$ for 20 minutes. Supernatants were collected and reduced thiols (RSH) were quantified with Ellman's reagent. Following incubation for 5 minutes at $37^{\circ} \mathrm{C}$, the absorbance of supernatants at $412 \mathrm{~nm}$ was determined. ${ }^{26,27}$ Calibration curves were obtained using GSH solutions of known concentration. Intracellular RSH levels were normalized to cellular protein content in milligrams.

\section{Migration assays}

Assays were performed using $8 \mu \mathrm{m}$ pore polycarbonate membrane transwells (diameter $6.5 \mathrm{~mm}$ ) (Corning, NY, USA). After coating the lower membrane surface with $100 \mu \mathrm{L}$ of fibronectin 
$2 \mu \mathrm{g} / \mathrm{mL}$, membranes were washed with PBS. Afterwards, transwells were immersed in RPMI medium supplemented with $10 \%$ FBS. Suspensions of $1.5 \times 10^{5}$ cells (B16F10, B16F10 ${ }_{\text {QDs- }}$ ${ }_{\text {GSH-4NAC }}$ or B16F10 ${ }_{\text {QDs-GSH-10NAC }}$ ) prepared in serum-free RPMI medium were seeded into the upper chamber of transwells. After 2 hours, inserts were collected and the cells present in the upper chamber were removed. Cells attached to the lower membrane surface after migration were fixed and stained with a $0.1 \%$ crystal violet solution in methanol $20 \%$ for 2 hours at $37^{\circ} \mathrm{C}$. Membranes were then gently washed, dried at room temperature, mounted in Mowiol, and observed under a light microscope. At least seven fields were evaluated per experiment to determine the number of transmigrated cells.

\section{Invasion assays}

Assays were performed using Matrigel Invasion Chambers ( $8 \mu \mathrm{m}$; Corning, NY, USA), which were rehydrated with RPMI media supplemented with 10\% FBS. Subsequently, suspensions of either B16F10 or B16F10 ${ }_{\text {QDs-GSH-10NAC }}$ cells $\left(2 \times 10^{5}\right)$ prepared in serum-free RPMI medium were seeded into the upper transwell chamber. After 24 hours, inserts were collected and cells remaining in the upper chamber were removed. Cells that invaded the matrigel and then attached to the lower membrane surface were fixed in cold methanol for 2 minutes and then stained with 1\% Toluidine Blue for 2 hours. Membranes were then gently washed, dried at room temperature, mounted in Mowiol, and observed under a light microscope. At least seven fields were evaluated per experiment to determine the number of cells that invaded the matrigel.

\section{rQDs-GSH cell labeling persistence and proliferation assay}

This assay was performed using $1 \times 10^{5}$ viable cells (B16F10 cells and B16F10 ${ }_{\text {QDs-GSH-10NAC }}$ ). Cells were seeded in $60 \mathrm{~mm}$ plates and cultured with RPMI-supplemented media. Afterwards, cells were detached with trypsin-EDTA $0.5 \%$ and centrifuged at $150 \times g$ for 5 minutes. Cell pellets were washed in PBS and centrifuged at $150 \times g$ for 5 minutes; the supernatants were discarded and cells were resuspended in $200 \mu \mathrm{L}$ of FBS. Then, the total number of cells 1-5 days post-inoculation was quantified in a Neubauer chamber (Marienfeld Superior, Lauda-Königshofen, Germany) after trypan blue staining $0.5 \%$ (biological industries). Percentage of viability, labeled viable cells, and MFI were determined by FACS, as previously described.

\section{In vivo metastasis assays}

Experiments were done essentially following previously described protocols. ${ }^{25}$ Briefly, C57BL/6 mice were injected intravenously with $2 \times 10^{5}$ B16F10 or B16F $10_{\text {QDs-GSH-10NAC }}$ cells in $500 \mu \mathrm{L}$ of physiological saline solution. Then, 21 days post-injection, mice were sacrificed. Lungs were fixed in Fekete's solution. Black lung tumor was separated from the rest of the lung tissue and weighed. Metastasis was expressed as black tissue mass/total lung mass percentage ( $\%)$ post-fixation.

\section{Mouse imaging}

C57BL/6 mice were injected intravenously with $2 \times 10^{5}$ $\mathrm{B} 16 \mathrm{~F} 10$ or B16F10 ${ }_{\mathrm{QDs}-\mathrm{GSH}-10 \mathrm{NAC}}$ cells, in $500 \mu \mathrm{L}$ of physiological saline solution. Mice were observed for 6 hours using the in vivo imaging system (IVIS; In-Vivo FX PRO; Bruker, Billerica, MA, USA). Excitation and emission wavelengths were 410 and $600 \mathrm{~nm}$, respectively. Afterwards, mice were euthanized and lungs were collected for ex-vivo imaging.

\section{In vivo imaging}

C57BL/6 mice were injected intravenously with $2 \times 10^{5}$ B $16 \mathrm{~F} 10_{\text {Control }}$ and B16F10 ${ }_{\text {QDs-GSH-10NAC }}$ cells in $500 \mu \mathrm{L}$ of physiological saline solution. Mice were sacrificed at 5 minutes, 30 minutes, 2 hours, 6 hours, and 24 hours postinjection. Lungs, heart, spleen, thymus, liver, and kidneys were harvested and fixed in Fekete's solution for 1 week. Fluorescence present at the different time points was quantified using IVIS, and are shown as the average of relative optical intensity (ROI). Fluorescence data are presented as percentages, considering the baseline fluorescence of unlabeled organs as $100 \%$.

\section{Immunohistochemical analysis}

The organs obtained from C57BL6 mice 6 and 24 hours after injection of B16F10 ${ }_{\text {QDs-GSH-10NAC }}$ B 16F10 ${ }_{\text {Calcein }}$, or B16 F10 ${ }_{\text {Control }}$ cells were fixed for 24 hours in $\mathrm{pH} 7$-buffered formalin (10\%) and then paraffin-embedded. For tissue samples, $5 \mu$ m-thick serial paraffin sections were collected on silanized slides (DAKO) and deparaffinized in two consecutive 5-minute incubations with xylene. One section was then stained with hematoxylin and eosin (H\&E). The others were rehydrated in two consecutive 3-minute incubations with $98 \%$ and $90 \%$ ethanol each, followed by a single incubation in Diethyl pyrocarbonate (DEPC)-treated distilled water for 5 minutes. Sections were then incubated in $2.5 \mu \mathrm{g} / \mathrm{mL}$ of Proteinase $\mathrm{K}$ (Invitrogen) at room temperature for 20 minutes and then rinsed twice for 3 minutes in DEPC-treated water. Nuclei were stained with DAPI for 5 minutes and washed. The samples were then mounted on cover slips and embedded with fluorescence mounting medium (DAKO). The images were captured using a fluorescence microscope (Olympus, Tokyo, Japan). 


\section{Proof-of-concept}

B16F10 cells stably transfected with either the empty pLacIOP vector [B16F10(Mock)] or the vector containing the Caveolin-1 (CAV1)-encoding insert [B16F10(CAV-1)] have been described previously. ${ }^{25,28}$ These cells were then labeled with rQDs-GSH as described earlier (B16F10 GSH-10NAC). C57BL/6 mice were injected intravenously with $2 \times 10^{5}$ of the respective cells in $500 \mu \mathrm{L}$ of physiological saline solution. Mice were sacrificed 6 hours post-injection of cells and lungs were fixed in Fekete's solution for 1 week. Then, fluorescence was quantified (average ROI) using the IVIS system.

\section{Statistical analysis}

Data shown are the average \pm SEM of at least three independent experiments. Statistical significance was determined at a 95\% CI using the nonparametric Mann-Whitney test, for the comparison of two groups.

All in vivo assays were performed as blind studies (in vivo metastasis assays and in vivo images) or double-blind studies (mice and lung images, immunohistochemistry assay, and proof-of-concept). All in vivo experiments were performed following the protocols of management and care of animals established by the CICUA of the University of Chile. CICUA approval code: CBA \# 0837 FMUCH.

\section{Results and discussion Synthesis and characterization of QDs-GSH}

In this study, green and red QDs-GSH were synthesized using the biomimetic method developed by our laboratory. ${ }^{8,9,22}$ Data summarizing the size distribution, zeta potential, and emission peak wavelengths of QDs-GSH are shown in Table 1. For gQDs-GSH, zeta potential values were greater than those of rQDs-GSH. GSH is a tripeptide, with a thiol and two carboxyl-terminal groups, which confer negative charges to the molecule. ${ }^{17}$ Thus, the elevated zeta potential is indicative of a higher GSH content in the gQDs-GSH. We

Table I Size (hydrodynamic diameter), Z potential, and emission peaks of green and rQDs-GSH

\begin{tabular}{l|l|l}
\hline & gQDs-GSH & rQDs-GSH \\
\hline Size $(\mathrm{nm})$ & $1.8 \pm 0.2$ & $5.1 \pm 1.1$ \\
$Z$ potential $(\mathrm{mV})$ & $-43.5 \pm 0.8$ & $-13.6 \pm 2.2$ \\
Emission peak $(\mathrm{nm})$ & 490 & 610 \\
\hline
\end{tabular}

Abbreviations: GSH, glutathione; gQDs-GSH, green QDs-GSH; QDs, quantum dots; rQDs-GSH, red QDs-GSH. previously characterized by X-ray photoelectron spectroscopy (XPS) the GSH-QD capping and reported a lower GSH content in rQDs-GSH than gQDs-GSH. ${ }^{23}$ Importantly, differences in QDs size (gQDs-GSH vs rQDs-GSH) and charge have previously been shown to affect cell viability. ${ }^{7,9,15,21,29}$ In addition, we have previously reported that green biomimetic QDs-GSH contain less $\mathrm{Cd}^{2+}$ than rQDs-GSH (18\% and 30\%, respectively). ${ }^{8,9}$ Therefore, in vitro assays were performed to evaluate cell behavior after incorporation of gQDs-GSH and rQDs-GSH.

\section{Characterization of QDs-GSH internalization and effects in BI6FIO cells}

B16F10 murine melanomas are cancer cells with an elevated metastatic potential that specifically accumulate in the lungs following tail vein injection of syngeneic C57BL/6 recipient animals, where they form readily detectable, pigmented metastatic nodules 15-21 days post-injection..$^{24,25}$ This is a well-established preclinical model that is employed to evaluate the metastatic potential of cancer cells. ${ }^{24,25}$ To label the B16F10 cells, they were incubated with gQDs-GSH or rQDs-GSH at concentrations ranging from 0 (control) to $50,100,200$, or $400 \mu \mathrm{g} / \mathrm{mL}$, in the presence of $4 \mu \mathrm{L} / \mathrm{mL}$ of lipofectamine, for 24 hours. Lipofectamine was used to promote cell internalization of QDs-GSH, following a previously described proto$\mathrm{col}^{8}$ (Patent No US 20130284979). ${ }^{22}$ Then, cell viability, percentage of viable labeled cells, and the MFI of cells transfected with different concentrations of QDs-GSH were determined by flow cytometry (Figure 1A). For gQDs-GSH, cell viability in general and viability of labeled cells were substantially reduced at concentrations of 50 $\mu \mathrm{g} / \mathrm{mL}$ or higher (Figure 1B). In this respect, rQDs-GSH were significantly less toxic and also the MFI for labeled cells was higher in comparison to those obtained with gQDs-GSH (Figure 1C and D). Note that rQDs-GSH only induced significant decreases in cell viability when used at higher concentrations $(400 \mu \mathrm{g} / \mathrm{mL})$. These observations are consistent with reports by Lovrić et al, ${ }^{30}$ who showed that smaller sized QDs more readily crossed intracellular membranes to enter the nucleus and other subcellular compartments, like mitochondria. ${ }^{8,31}$ The nuclear pore complex permits entry of small molecules up to $\sim 9 \mathrm{~nm}$ in diameter by passive diffusion..$^{32}$ Our results show that gQDs-GSH and rQDs-GSH possess a hydrodynamic diameter of $\sim 1.8$ and $\sim 5.1 \mathrm{~nm}$ (Table 1), respectively, indicating that both QDsGSH probably can cross the nuclear membrane, although gQDs-GSH are likely do so more efficiently. However, QDs toxicity may also relate to the differences in zeta potential 
A

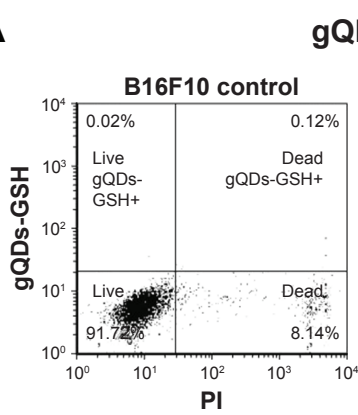

gQDs-GSH
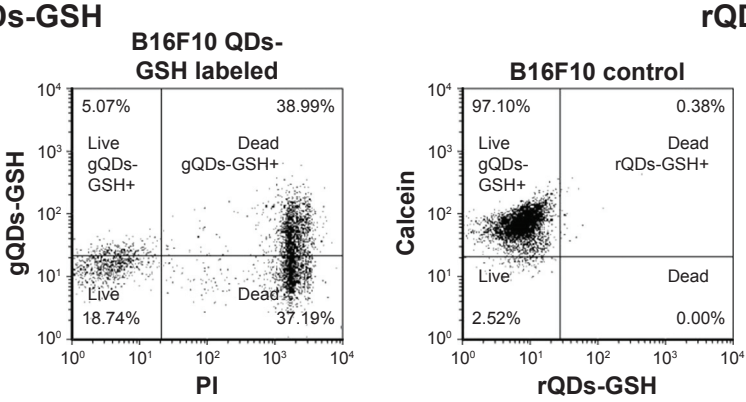

rQDs-GSH

B

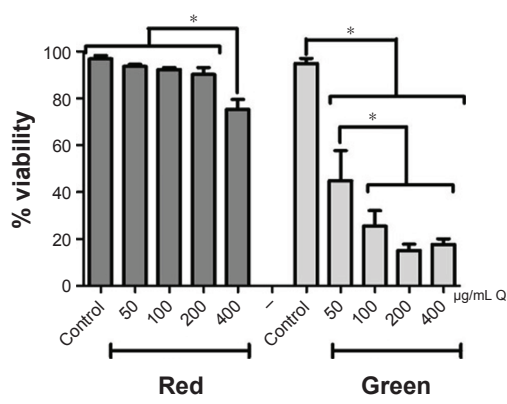

C

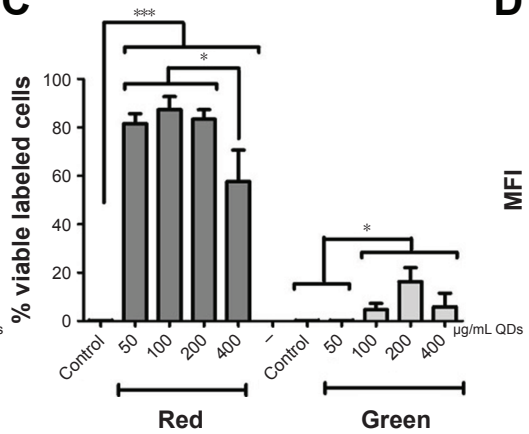

D
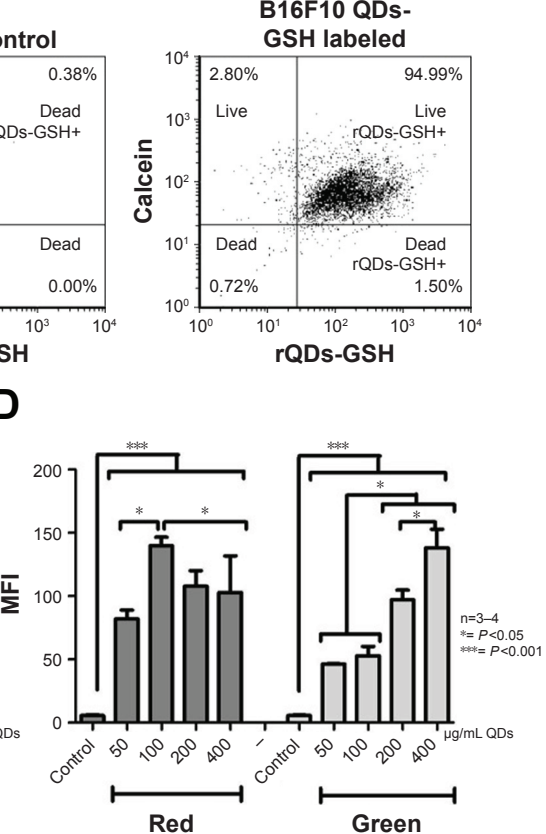

Figure I BI6FIO cells viability after rQDs-GSH and gQDs-GSH incorporation.

Notes: (A) Dot plot of BI6FI0 cells labeled with gQDs-GSH or rQDs-GSH (I00 $\mu \mathrm{g} / \mathrm{mL})$, and BI6FI0 control cells. Events were gated to show BI6FI0 gQDs-GSH+ or rQDs-GSH+ cells and live or dead BI6FI0 cells. (B) Effects on BI6FI0 cell viability of treatments with lipofectamine and gQDS-GSH or rQDs-GSH. (C) Comparison of viable labeled cells treated with either rQDs-GSH or gQDs-GSH, and lipofectamine. (D) Comparison of MFI associated with either rQDs-GSH or gQDs-GSH in viable BI6FI0 cells. Results were averaged from 3 to 4 independent experiments $(n=3-4)$. Data were analyzed using the nonparametric Mann-Whitney test. Statistically significant differences compared with the controls and different treatments are indicated.

Abbreviations: GSH, glutathione; gQDs-GSH, green QDs-GSH; MFI, mean fluorescence intensity; QDs, quantum dots; rQDs-GSH, red QDs-GSH.

between gQDs-GSH and rQDs-GSH, given that greater negative zeta potential values, as is the case for gQDs-GSH, are known to reduce cell survival ${ }^{31}$ (Table 1).

Of note, the detrimental effects observed in B16F10 cells incubated with gQDs-GSH were significantly elevated in comparison to the toxic effects of biomimetic QDs previously reported for MKN45 cells. ${ }^{8,9,23}$ This may simply reflect cellspecific parameters of predisposition to QDs-GSH toxicity, as has been reported previously in different cell lines exposed to inorganic NPs. ${ }^{33}$ Alternatively, it is well established that the generation of ROS represents one of the main mechanisms by which QDs induce damage to biological systems. ${ }^{13-15,19}$ Intracellular thiols (RSH) play an important role as mediators of tolerance to intracellular ROS. ${ }^{17,18}$ Thus, we hypothesized that the differences in sensitivity to QDs-GSH observed for B16F10 and MKN45 cells may be due to different levels of RSH. To evaluate this possibility, intracellular RSH levels were determined in each cell type. As suspected, significantly lower RSH levels were observed in B16F10 cells than in MKN45 cells, suggesting that B16F10 cells may, for this reason, tolerate to a lesser extent QD-induced oxidative damage than MKN45 cells (Figure S2).

\section{NAC treatment reduces rQDs-GSH effects in BI6FIO cells and increases cell labeling}

QDs increase ROS in biological systems, ${ }^{14}$ and ROS, on the other hand, affects migration and invasion of metastatic cells. ${ }^{34}$ Therefore, we hypothesized that NAC should ameliorate detrimental QDs-GSH effects on metastatic cells, given that NAC increases intracellular RSH levels. ${ }^{17}$ Moreover, NAC is generally beneficial and protects cells against organic stress induced by inorganic NPs, ${ }^{33}$ as well as provides greater protection against QDs damage than other non-thiol anti-oxidants, such as trolox. ${ }^{30}$ Given our results showing that incubation of cells with $100 \mu \mathrm{g} / \mathrm{mL}$ of rQDs-GSH resulted in $>80 \%$ viable labeled cells, similar to the percentage of viable labeled cells reported by Voura et al, ${ }^{12}$ with the best mean MFI values (Figure 1C and D), as well as the fact that fluorescence emission at longer wavelengths is absorbed to a lesser extent by tissues, ${ }^{4}$ we focused on characterizing the behavior of B16F10 cells after labeling with $100 \mu \mathrm{g} / \mathrm{mL}$ rQDs-GSH.

To assess the benefits of NAC, B16F10 cells were cultured as previously mentioned, but this time different concentrations of NAC were added in both culture steps (Figure 2A). Afterwards, cell viability, ROS levels, viable 
A

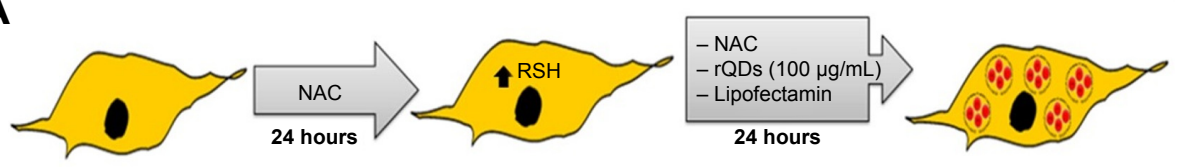

B

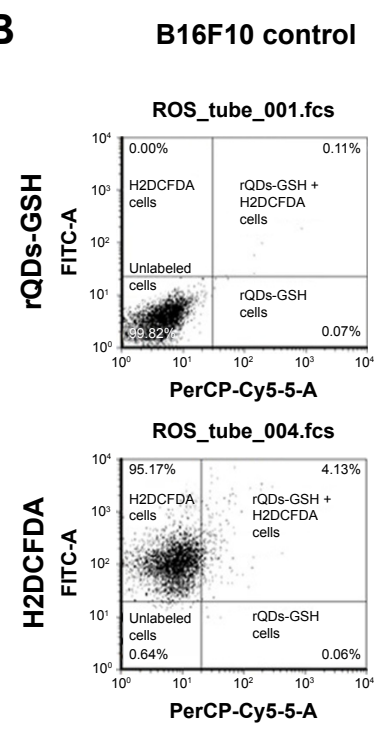

D

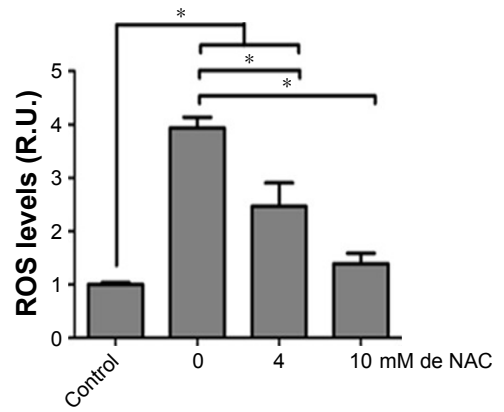

B16F10 QDsGSH labeled

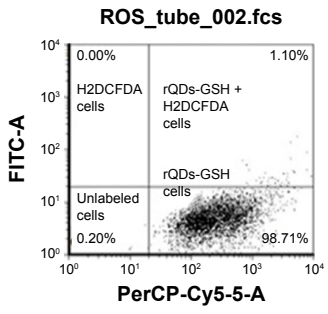

ROS tube 012.fcs

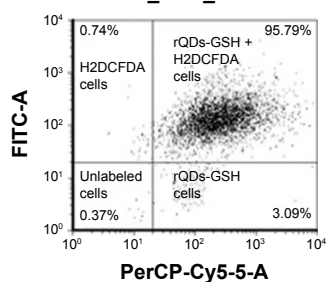

$\mathbf{E}$

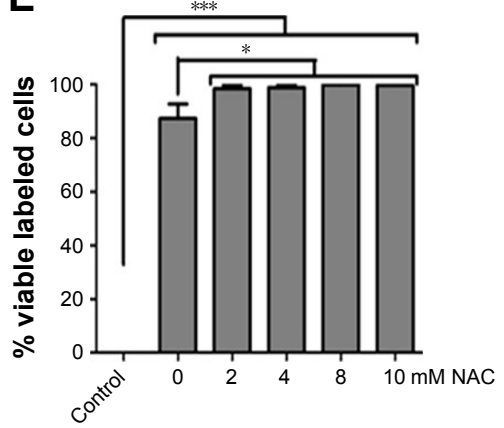

Calcein
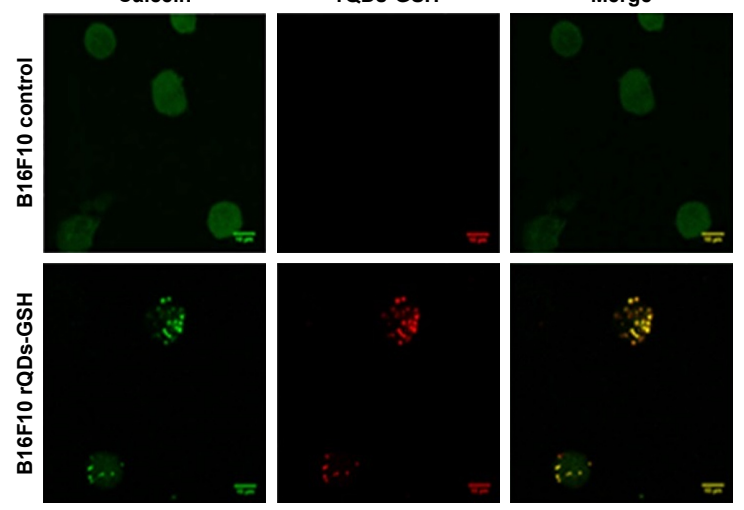

$\mathbf{F}$

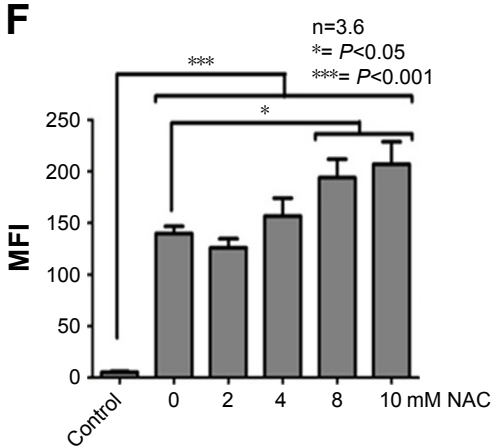

Figure 2 Effects of NAC treatment on ROS levels, viability, and incorporation of rQDs-GSH into BI6FI0 cells.

Notes: (A) Schematic summarizing the labeling assays in the presence of NAC. (B) Dot plot of BI6FI0 cells labeled with H2DCFDA or rQDs-GSH (I00 $\mu$ g/mL) and BI6FI0 control cells. Events were gated to show BI6FIO H2DCFDA+ or rQDs-GSH+ BI6FI0 cells. (C) Confocal imaging of viable BI6FI0 cells (Calcein labeled) with or without rQDs-GSH. (D) ROS levels of BI6FI0 cells labeled with $100 \mu \mathrm{g} / \mathrm{mL}$ of rQDs-GSH in the presence of 0 , 4, or $10 \mathrm{mM}$ NAC during the labeling process. (E) Percentage of viable rQDs-GSH-labeled BI6FI0 cells in the presence of different NAC concentrations. (F) MFI associated with QDs-GSH fluorescence in viable rQDs-GSH-labeled BI6FI0 cells in the presence of different NAC concentrations. Results were averaged from 3 to 6 independent experiments $(n=3-6)$. Data were analyzed using the nonparametric Mann-Whitney test. Statistically significant differences compared with the controls and different treatments are indicated.

Abbreviations: GSH, glutathione; H2DCFDA, dichlorodihydrofluorescein; hrs, hours; MFI, mean fluorescence intensity; NAC, N-acetylcysteine; QDs, quantum dots; rQDs-GSH, red QDs-GSH.

labeled cells, and MFI associated with QDs-GSH presence in viable cells were detected by flow cytometry (Figure $2 \mathrm{~B}$ ). rQDs-GSH were incorporated by B16F10 cells and intracellular distribution was similar (Figure 2C) to that described in previous studies. ${ }^{8,9,23}$ ROS levels were lower when cells were co-incubated with rQDs-GSH in the presence of NAC (Figure 2D). Nearly 100\% viable labeled B16F10 cells were obtained when incubated with $10 \mathrm{mM}$ NAC (Figure 2E). Also, a general increase in MFI values after exposure to 8 and 10 mM NAC was observed (Figure 2F). Because fluorescence is proportional to the QDs-GSH concentration, ${ }^{8}$ this suggests that addition of NAC to cell cultures promotes QDs-GSH internalization, possibly by reducing ROS formation upon QDs uptake. ${ }^{30}$ Finally, NAC treatments allowed obtaining nearly $100 \%$ viable labeled cells with increased fluorescence signal and ROS levels similar to unlabeled cells.

\section{Effects of rQDs-GSH and NAC treatment on functional parameters of BI6FIO cells}

To compare NAC effects, cells were incubated with $100 \mu \mathrm{g} / \mathrm{mL}$ of rQDs-GSH in the presence of either 4 (maximum NAC concentration that did not significantly increase the MFI) or $10 \mathrm{mM}$ NAC (upper limit NAC concentration used for 

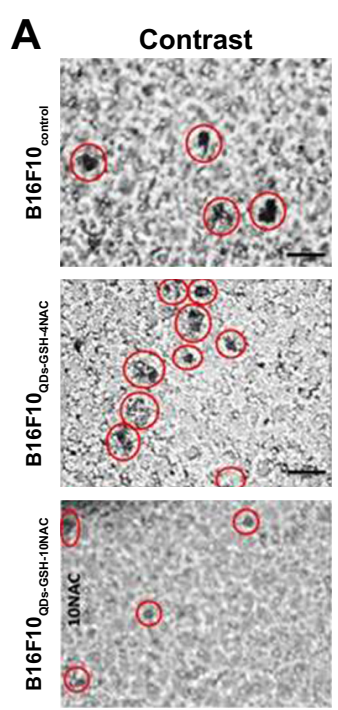

C
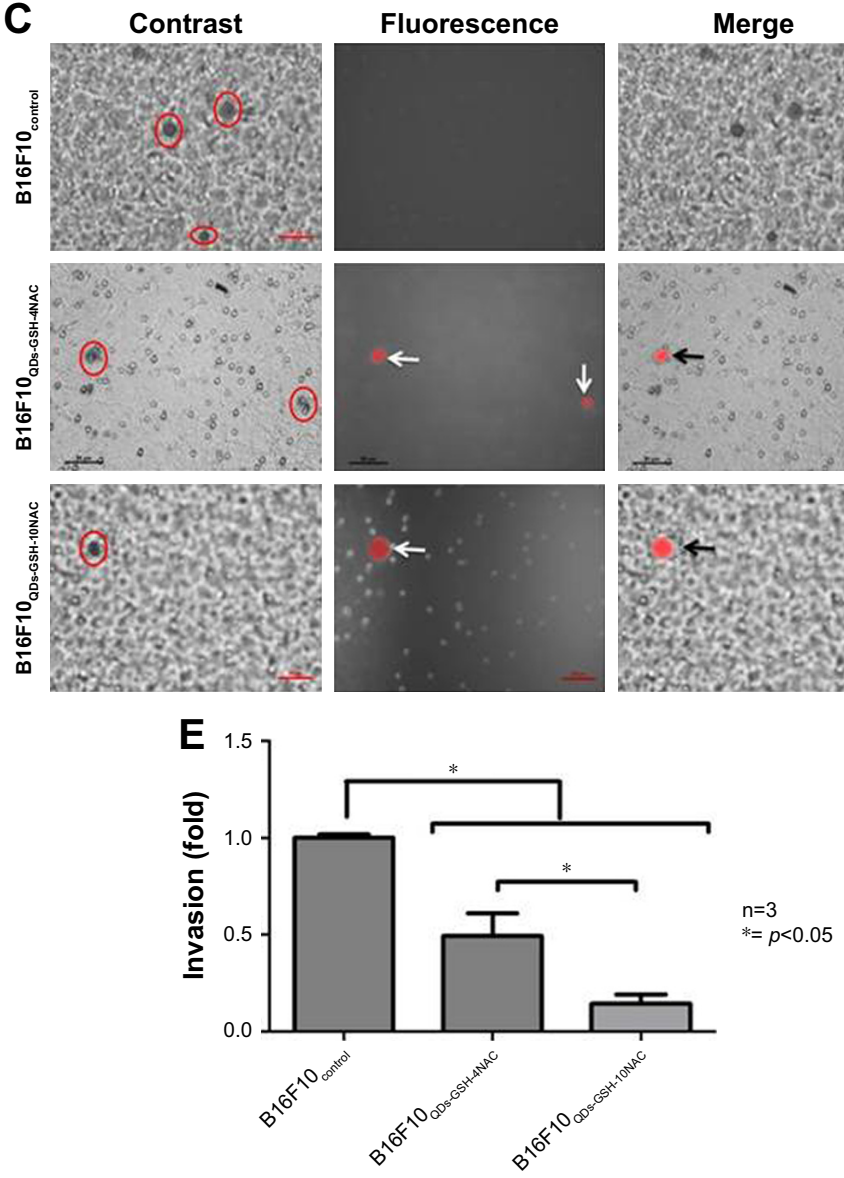

B

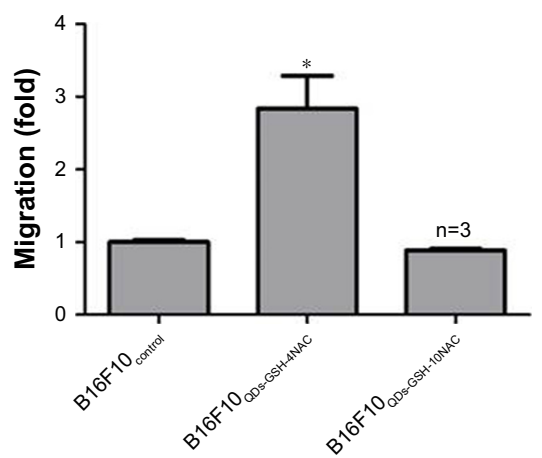

D B16F10 ${ }_{\mathrm{QDS}-\mathrm{GSH}-10 \mathrm{NAC}}$
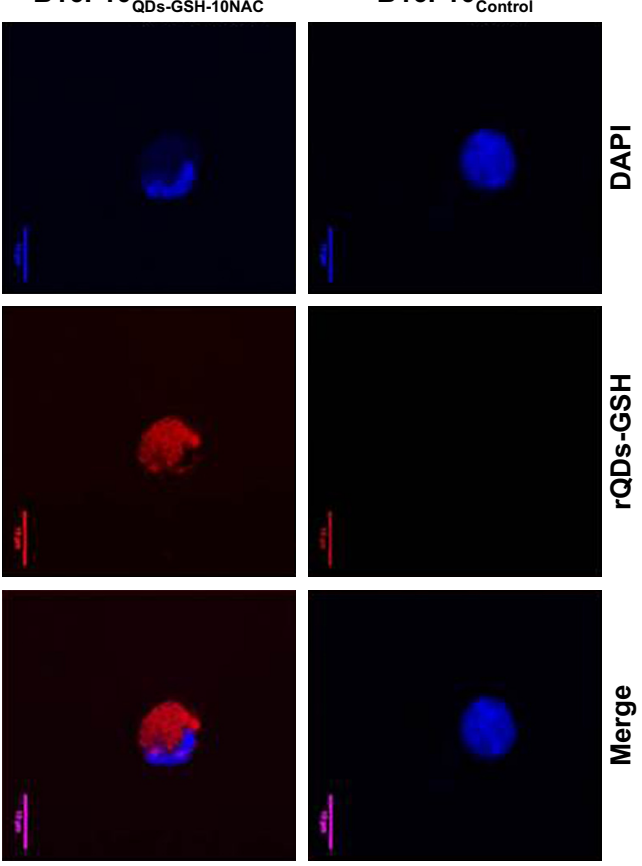

Figure 3 Migration and invasion of BI6FI0 cells labeled with rQDs-GSH in the presence of NAC.

Notes: (A) Images obtained by epifluorescence optical microscopy of migratory BI6FI0 control, BI6FI0 ${ }_{\mathrm{QDs}-G S H-4 N A C}$, and BI6FI0 ${ }_{\mathrm{QDs}-\mathrm{GSH}-4 \mathrm{NAC}}$ cells. In circles, migratory cells; white arrows: fluorescent cells; black arrows: migratory rQDs-GSH-labeled cells. (B) Quantification of the migration of $\mathrm{BI}_{6 \mathrm{FIO}} \mathrm{QDD}_{\mathrm{QSH}-4 \mathrm{NAC}}, \mathrm{BI}^{6 \mathrm{FIO}}{ }_{\mathrm{QDS}-\mathrm{GSH}-10 \mathrm{NAC}}$, and control cells. (C) Images obtained by epifluorescence optical microscopy of invading BI6FIO control, BI6FIO ${ }_{\mathrm{QDs}-G S H-4 N A C}$, and BI6FIO ${ }_{\mathrm{QDs}-G S H-4 N A C}$ cells. In circles, migratory cells; white arrows: fluorescent cells; black arrows: migratory rQDs-GSH-labeled cells. (D) Confocal microscopy of invading BI6FI0 the invasiveness of $\mathrm{BI} 6 \mathrm{FIO}{ }_{\mathrm{QDs}-G S H-4 N A C}, \mathrm{BI} 6 \mathrm{FIO}{ }_{\mathrm{QDs}-\mathrm{GSH}-10 \mathrm{NAC}}$, and control cells. Results were averaged from three independent experiments ( $\mathrm{n}=3$ ). Data were analyzed using the nonparametric Mann-Whitney test. Statistically significant differences compared with the controls and different treatments are indicated.

Abbreviations: $\mathrm{BI} 6 \mathrm{FIO}{ }_{\mathrm{QDs}-G S H-4 N A C}, \mathrm{BI} 6 \mathrm{FIO}$ cells labeled with rQDs-GSHin presence of $4 \mathrm{mM}$ of NAC; BI6FI0 ${ }_{\mathrm{QDs}-G S H-10 \mathrm{NAC}}$, BI6FI0 cells labeled with rQDs-GSH in presence of $10 \mathrm{mM}$ of NAC; GSH, glutathione; NAC, N-acetylcysteine; QDs, quantum dots; rQDs-GSH, red QDs-GSH.

treatment) to generate $\mathrm{B} 16 \mathrm{~F} 10_{\mathrm{QDs}-\mathrm{GSH}-4 \mathrm{NAC}}$ and $\mathrm{B} 16 \mathrm{~F} 10_{\mathrm{QDs}-}$ GSH-10NAC, respectively. Subsequently, cells were evaluated in vitro in migration and invasion assays.
Migration was 2.8 times higher for B16F10 ${ }_{\text {QDs-GSH-4NAC }}$ than control cells (Figure 3A and B). On the other hand, no differences in migration were observed when $\mathrm{B} 16 \mathrm{~F} 10_{\text {QDs-GSH-10NAC }}$ 
cells were compared with control cells. This suggests that B16F10 cells with internalized rQDs-GSH increase migration at lower NAC concentrations, likely as the consequence of increased intracellular ROS levels (Figure 2D) that are known to promote cancer cell migration. ${ }^{34}$ Therefore, NAC pretreatment permitted ameliorating undesirable effects observed following QDs-GSH internalization related to intracellular ROS levels, and allowed obtaining QDs-GSH-labeled B16F10 cells that migrated like the unlabeled cells.

Likewise, rQDs-GSH-labeled B16F10 cells were still able to invade in a matrigel invasion assay (Figure 3C), maintaining the labeling associated with QDs-GSHr (Figure 3C and D), but did so much less effectively. rQDs-GSH presence significantly decreased invasion for both B16F10 ${ }_{\text {QDs-GSH-4NAC }}$ and B16F10QD$\mathrm{s}_{\mathrm{QDS}-\mathrm{GSH}-10 \mathrm{NAC}}$, to $41 \%$ and $6 \%$, respectively (Figure 3E). These results suggest that greater amounts of internalized rQDs-GSH (Figure 2F) correlate with a reduction in cell invasiveness. Thus, because augmenting intracellular ROS levels increases cell metastasis, ${ }^{34}$ our results indicate that internalization of rQDs-GSH in B16F10 cells decreases invasiveness in a ROSindependent fashion. A possible explanation might be that $\mathrm{Cd}^{2+}$ in rQDS-GSH reduces the activity of matrix metalloproteinases (MMPs), which are zinc-containing endopeptidases capable of degrading extracellular matrix (ECM) proteins. ${ }^{35}$ MMPs are exocytosed by metastatic cells and mediate tumor microenvironment changes during cancer progression, favoring metastatic cell invasion. ${ }^{36} \mathrm{~B} 16 \mathrm{~F} 10$ exposure to $\mathrm{Cd}^{+2}$ has been shown to reduce cell invasiveness due to upregulation of tissue inhibitor of metalloproteinase-1 (TIMP-1) expression, which is $\mathrm{Cd}^{+2}$-dependent. ${ }^{37}$ In addition, our results show that the methodology employed here permits obtaining rQDs-GSH-labeled B16F10 cells that migrate similarly to unlabeled cells, but display dramatically reduced invasiveness. These results suggest that the invasiveness of rQDs-GSH-labeled cells is reduced by a cadmium-dependent mechanism, but further experimentation beyond the scope of this study is required to confirm this possibility.

Therefore, rQDs-GSH-labeled cells can be useful for in vivo studies of metastasis steps where migration is relevant (ie, distribution of metastatic cells throughout the body and transendothelial migration). With this in mind, we envisioned that $\mathrm{B} 16 \mathrm{~F} 10_{\text {QDS-GSH-10NAC }}$ cells might be useful to monitor early steps in metastasis. To this end, viability, proliferation, and QD-clearance were evaluated for 5 days after labeling using B16F10 ${ }_{\text {QDS-GSH-10NAC }}$ cells.

\section{Proliferation of $\mathrm{BI} 6 \mathrm{FIO}{ }_{\text {QDS-GSH-IONAC }}$ and persistence of labeling}

Intracellular NP content may be reduced by different mechanisms, such as cell death, cell proliferation, NP diffusion, lysosomal degradation, and/or transcytosis and exocytosis. ${ }^{38}$ For example, D-penicillamine-coated QDs (DPA-QDs) were not degraded in the lysosomal compartment during the first 24 hours post-internalization, ${ }^{39}$ but rather were rapidly exocytosed (half-life of 21 minutes). Therefore, it was important here to determine rQD-GSH persistence in $\mathrm{B} 16 \mathrm{~F} 10$ cells prior to the subsequent evaluation in in vivo metastasis assays. B16F10 ${ }_{\text {QDs-GSH-10NAC }}$ cells were cultured in RPMI supplemented with $10 \%$ BFS for 5 days and the total cell number (quantification of proliferation), cell viability, percentage of viable labeled cells, and MFI were determined after cell labeling (Figure 4). B16F10 ${ }_{\text {QDs-GSH-10NAC }}$ viability was not substantially reduced during a 5 -day period postlabeling (Figure 4A), although a slight decline in viability was observed after the third day (to 79\%), which then remained stable up to day 5. A significant reduction in MFI was observed from the second day onwards, which resulted in a reduction of the fluorescence intensity by $43 \%$ after 5 days (Figure 4C). The data obtained suggest that B16F $10_{\text {QDs-GSH-10NAC }}$ cells remain viable with a stable percentage of viable labeled cells for at least 3 days (Figure 4B), and that the MFI does not vary significantly until the second-day post-labeling. Thus, the labeling methodology described here employing biomimetic QDs-GSH allows for more prolonged cell labeling than other QDs-based methodologies described in the literature (on the order of minutes). ${ }^{39}$ Interestingly, B16F10 ${ }_{\text {QDs-GSH-10NAC }}$ cells appear unable to proliferate, since no increase in the total number of cells was observed during the 5 days of the assay (Figure 4D). For rQDs-GSH-labeled $\mathrm{B} 16 \mathrm{~F} 10$ cells, in the absence of NAC treatments (lower case labeling in Figure 2), the total number of cells was found to increase 24 hours post-labeling in a manner similar to unlabeled cells (Figure S3D), whereby viability, percentage of labeled cells, and MFI were maintained (Figure S3A-C). These results are in agreement with previous publications indicating that NP internalization may affect cellular proliferation ${ }^{40}$ and that $\mathrm{Cd}^{2+}$ inhibits cell proliferation in a dosedependent manner. ${ }^{41,42}$ Thus, the methodology described here has the potential to be used as a cell-labeling strategy to study early steps in vivo involved in accumulation/distribution of metastatic cells in distant organs.

\section{In vivo metastasis assays}

For the in vivo studies, we employed a syngeneic metastasis model in C57BL/6 mice with an intact immune system. $\mathrm{B} 16 \mathrm{~F} 10_{\mathrm{QDs}-\mathrm{GSH}-10 \mathrm{NAC}}$ cells were injected into the tail vein of mice and 21 days post-injection mouse survival and tumor mass in the lungs were determined (Figure 5A). Administration of B16F10 ${ }_{\text {QDs-GSH-10NAC }}$ cells did not alter mouse survival 

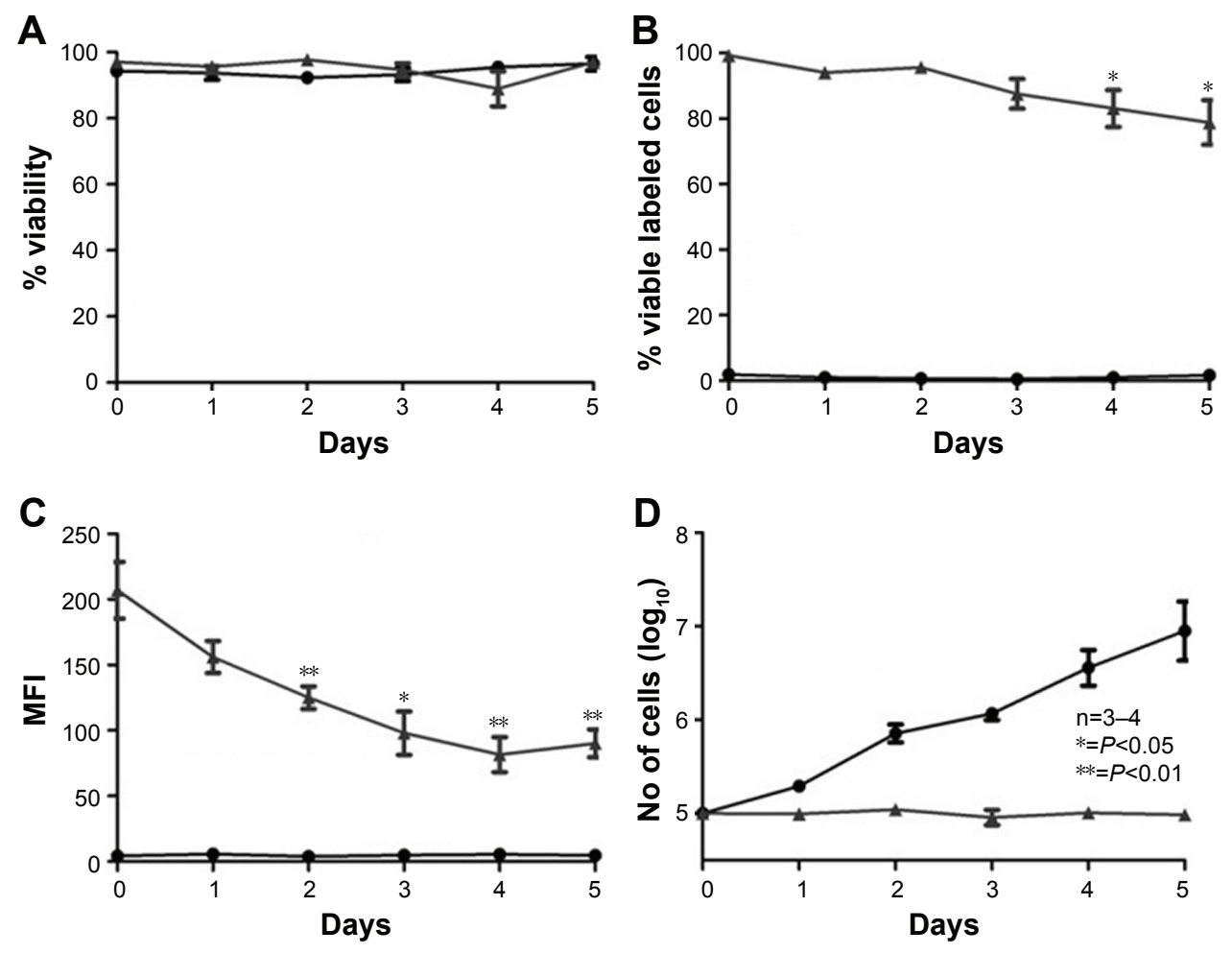

$$
\rightarrow \text { B16F10 }
$$

Figure 4 Effects of QDs-GSH labeling on BI6FIO cell proliferation and QDs-GSH signal persistence.

Notes: (A) BI6FIO ${ }_{\mathrm{ODs}-G S H-I O N A C}$ and control cell viability. (B) Percentage of viable BI6FIO ${ }_{\mathrm{QDs}-G S H-10 \mathrm{NAC}}$ cells. (C) MFI of viable BI6FI0 $\mathrm{BI} 6 \mathrm{FIO}_{\mathrm{QDS}-\mathrm{GSH}-10 \mathrm{NAC}}$ cells. Results were obtained over a 5-day period after labeling and averaged from 3 to 6 independent experiments ( $\mathrm{n}=3-6$ ). Data were analyzed using the nonparametric Mann-Whitney test. Statistically significant differences compared with the controls and different treatments are indicated.

Abbreviations: $\mathrm{BI}_{6 \mathrm{FIO}}{ }_{\mathrm{QDs}-\mathrm{GSH}-10 \mathrm{NAC}}, \mathrm{BI6FI0}$ cells labeled with $\mathrm{rQDs}-\mathrm{GSH}$ in presence of $10 \mathrm{mM}$ of NAC; GSH, glutathione; MFI, mean fluorescence intensity; NAC, $\mathrm{N}$-acetylcysteine; QDs, quantum dots; rQDs-GSH, red QDs-GSH.
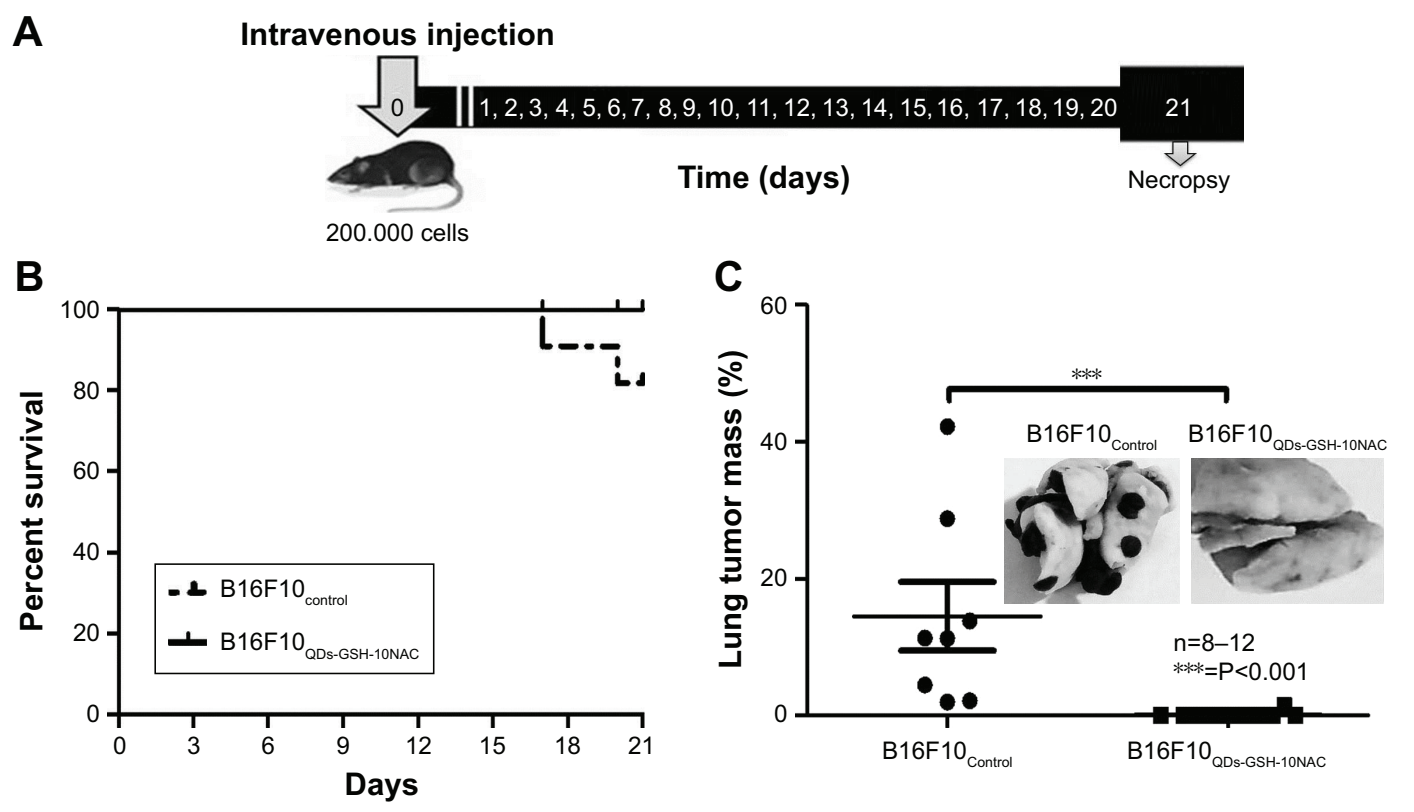

Figure 5 In vivo metastasis assays using rQDs-GSH-labeled BI6FI0 cells.

Notes: $\mathrm{BI6FIO}{ }_{\mathrm{QDs}-G S H-10 N A C}$ and control cells were injected into the tail vein of C57BL/6 mice. After 21 days, mice were sacrificed and lungs were collected. (A) Scheme summarizing the time course of metastasis assays. (B) C57BL/6 mouse survival up to 21 days post-injection of BI6FI0 (dashed line) or BI6FI0 ${ }_{\mathrm{QDs}-G S H-I 0 N A C}$ (solid line) cells. (C) Determination of tumor mass present in lungs from C57BL/6 mice at 21 days post-injection of BI6FI0 control or BI6FI0 8 (BI6FIO control cells) or 12 (BI6FI0 ${ }_{\text {QDs-GSH-IONAC }}$ cells) independent experiments in mice $(\mathrm{n}=8-12)$. Data were analyzed using the nonparametric Mann-Whitney test. Statistically significant differences compared with the controls and different treatments are indicated. Note that the data shown were obtained in a double-blind study. Abbreviations: $\mathrm{BI}_{6 \mathrm{FIO}} \mathrm{QDs}-\mathrm{GSH}-\mathrm{IONAC}, \mathrm{BI6FI0}$ cells labeled with rQDs-GSH in presence of $10 \mathrm{mM}$ of NAC; GSH, glutathione; NAC, N-acetylcysteine; QDs, quantum dots; rQDs-GSH, red QDs-GSH. 
(Figure 5B). After necropsy, metastasis remained undetectable in over $90 \%$ of the $\mathrm{B} 16 \mathrm{~F} 10_{\text {QDs-GSH-10NAC }}$ treated mice; lung tumor mass in the only mouse with metastasis was equivalent to only $1.4 \%$ of total lung mass. To the contrary, metastatic lung nodules were detected in all control mice injected with the same number of B16F10 cells (Figure 5C) and accounted for $14.5 \%$ of lung mass, similar to what we have described in our previous publications. ${ }^{25,28}$ These results show that incorporating QDs into B16F10 cells, as is the case for B16F10 ${ }_{\mathrm{QDS}-\mathrm{GSH}-10 \mathrm{NAC}}$, significantly reduces the ability of these cells to form metastatic nodules in vivo 21 days postinjection. This is in agreement with the in vitro data showing that labeled cells neither invade nor proliferate (Figures 3E and 4D). On the other hand, the use of B16F10 cells labeled with $\mathrm{CdSe} / \mathrm{ZnS}$ QDs capped with dihydrolipoic acid (DHLA) was reported using lipofectamine-aided transfection for internalization of QDs-DHLA. However, in those experiments, the authors observed that B16F10 cells formed metastatic nodules 40 days post-injection. ${ }^{12}$ These differences may be attributable to variations in the amount of QDs-GSH present and $\mathrm{Cd}^{2+}$ release inside B16F10 ${ }_{\mathrm{QDs}-\mathrm{GSH}-10 \mathrm{NAC}}$ cells. Thus, using our methodology, QDs-GSH-labeled cells were obtained that migrated similarly to unlabeled cells, but were unable to invade and proliferate.

Over the last 30 years, the repertoire of compounds containing transition metals with anti-tumor properties has expanded to include compounds containing $\mathrm{Zn}, \mathrm{Co}, \mathrm{Ni}, \mathrm{Sn}$, $\mathrm{Ag}$, and $\mathrm{Cd}^{43-49}$ Among these elements, cadmium has been ascribed elevated anti-tumor effects in comparison with other transition metals..$^{43}$ In 2016, Sreekanth et al reported that cadmium oxide $(\mathrm{CdO})$ NPs inhibited the proliferation of normal and tumor cell lines, whereby the tumor cells were more severely affected by CdO NPs. ${ }^{44}$ Our study shows that QDs-GSH have potent anti-tumor effects, as evidenced by the dramatic inhibition of cell proliferation and invasion following internalization of QDs-GSH. Moreover, the ability of such QD-GSH-labeled cells to form metastatic nodules in $\mathrm{C} 57 \mathrm{BL} / 6$ mice was ablated within the time frame analyzed. Data from the literature favor the notion that these anti-metastatic effects are related to the presence of cadmium in the QDs-GSH core. Our results highlight the need to further investigate the anti-tumor effects of QDs-GSH, bearing in mind that selective tumor accumulation should permit using these QDs-GSH both as diagnostic agents and in anti-tumor therapy (theranostics).

Here, however, we focused on evaluating the utility of B16F $10_{\text {QDs-GSH-10NAC }}$ in metastatic cells tracking at early stages post-injection, since they were able to migrate, but did not invade or proliferate.

\section{In vivo distribution of $\mathrm{BI} 6 \mathrm{FIO} 0_{\text {QDs-GSH-IONAC }}$ cells}

Previously, studies have shown that QDs can be followed by fluorescence microscopy in vitro and also to track interactions between different cell populations in vivo. ${ }^{12,50}$ For instance, this approach has permitted visualizing the clogging of blood vessels produced by B16-BL6 melanoma cells during metastasis. ${ }^{45}$ However, it was still not possible to track the accumulation or distribution of metastatic cells by in vivo fluorescence imaging.

To assess the potential utility of B16F10 $0_{\text {QDs-GSH-10NAC }}$ cells in recording early steps of metastasis by in vivo fluorescence imaging, cells were injected (Figure 6A and $\mathrm{B}$ ) into the tail vein of $\mathrm{C} 57 \mathrm{BL} / 6$ mice and whole animal images were obtained using the IVIS. After injection of the cells into mice, the fluorescence signal was not detectable (Figure S4), likely because a number of biological molecules, including hemoglobin, absorb light between 400 and $600 \mathrm{~nm}$, thereby interfering with the B16F10 ${ }_{\text {QDs-GSH-10NAC }}$ signal. Thus, animals were sacrificed and after necropsy at the indicated time points, organs were placed in Fekete's solution to eliminate blood traces and then fixed as described. ${ }^{46}$ Examples of lungs from C57BL/ 6 mice obtained after 6 hours inoculation with B16F10 ${ }_{\text {QDs-GSH-10NAC }}$ cells are shown (Figure 6C). Fluorescence due to $\mathrm{B} 16 \mathrm{~F} 10_{\mathrm{QDs}-\mathrm{GSH}-10 \mathrm{NAC}}$ presence in lungs was clearly distinguishable from the background fluorescence of controls (Figure 6D), suggesting the presence of B16F10 ${ }_{\text {QDs-GSH-10NAC }}$ in the lungs of $\mathrm{C} 57 \mathrm{BL} / 6$ mice.

Using this approach, we determined the kinetics of B16F10 accumulation, particularly in the lungs of C57BL/6 mice. To this end, $2 \times 10^{5} \mathrm{~B} 16 \mathrm{~F} 10_{\mathrm{QDs}-\mathrm{GSH}-10 \mathrm{NAC}}$ cells were injected into the tail vein of mice and then the following organs were analyzed: 1) lungs (metastatic cell target); 2) spleen, liver, and kidneys (principal QD storage organs post-intravenous injection) and; 3 ) heart and thymus (organs where no extensive accumulation of QDs has been previously described). ${ }^{47,48}$ Mice were sacrificed and organs were harvested at 5 minutes, 30 minutes, 2 hours, 6 hours, and 24 hours post-injection, and the fluorescence was detected by direct imaging of the organs (Figure $6 \mathrm{E}-\mathrm{J}$ ). The greatest difference was observed in lungs harvested 6 hours after B16F10 ${ }_{\text {QDs-GSH-10NAC }}$ injection (Figure 6E). Although a significant increase in fluorescence was detectable as soon as 5 minutes post-injection, the intensity increased until reaching a maximum 6 hours post-injection. B16F10 cells preferentially metastasize to the lungs of C57BL/6 mice, possibly due to their preference for laminin and/or fibronectin, two ECM proteins present surrounding small vessels of the lung, 
A

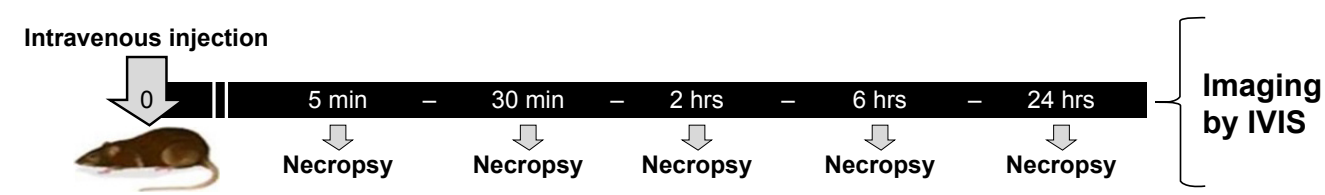
200.000 cells
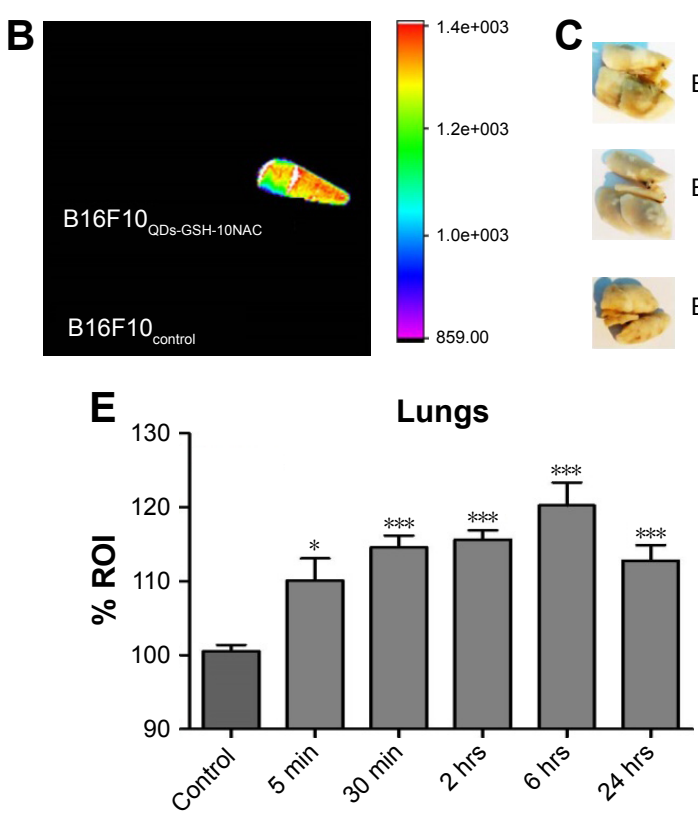

G
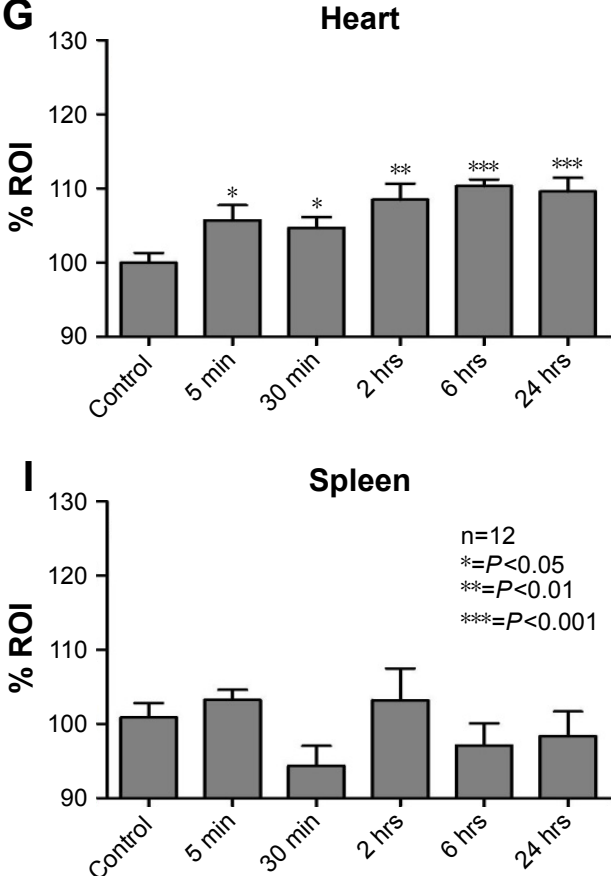

C

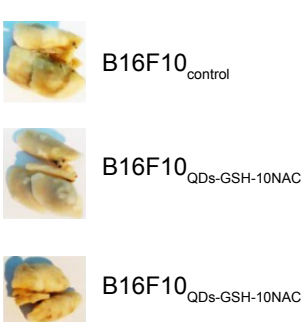

F

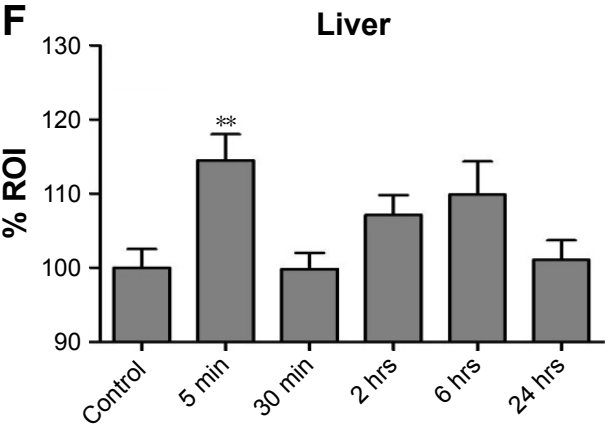

H

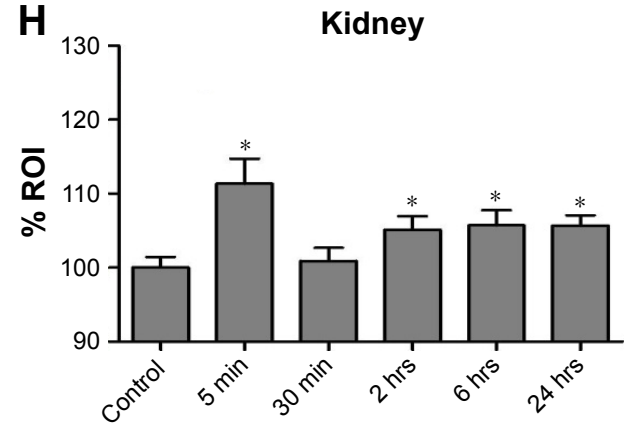

Thymus

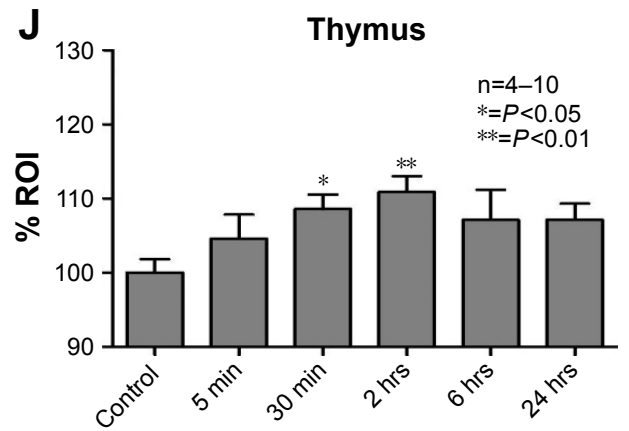

Figure 6 In vivo $\mathrm{BI} 6 \mathrm{FIO}{ }_{\mathrm{QDs}-G S H-10 N A C}$ cell tracking.

Notes: $\mathrm{BI} 6 \mathrm{FIO}{ }_{\mathrm{ODs}-\mathrm{GSH}-10 \mathrm{NAC}}$ cells were injected into the tail vein of C57BL/6 mice. rQDs-GSH signals were followed and quantified by imaging the signal in lungs. Organs were collected at 5 minutes, 30 minutes, 2 hours, 6 hours, and 24 hours post-injection. (A) A scheme summarizing the time course of early metastasis assays. (B) Eppendorf tubes containing BI6FIO control and BI6FIO ${ }_{\mathrm{QDS}-G S H-10 \mathrm{NAC}}$ cells used for injection in C57BL/6 mice. (C) Image showing lungs collected from C57BL/6 mice 6 hours post-injection with either control or labeled cells (duplicate). (D) Fluorescence images of lungs collected from C57BL/6 mice 6 hours post-injection with either control or labeled cells. (E-J) Quantification of rQDs-GSH-associated fluorescence in lungs, heart, kidneys, liver, spleen, and thymus of C57BL/6 mice at different time points after injection of

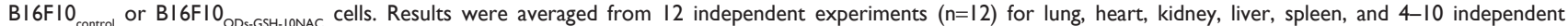
experiments $(n=4-10)$ for thymus. Data were analyzed using the nonparametric Mann-Whitney test. Statistically significant differences compared with the controls and different treatments are indicated. Note that the data shown were obtained in a double-blind study.

Abbreviations: $\mathrm{BI} 6 \mathrm{FIO}_{\mathrm{QDs}-\mathrm{GSH}-10 \mathrm{NAC}}, \mathrm{BI} 6 \mathrm{FI} 0$ cells labeled with rQDs-GSH in presence of $10 \mathrm{mM}$ of NAC; GSH, glutathione; IVIS, in vivo imaging system; NAC, N-acetylcysteine; QDs, quantum dots; rQDs-GSH, red QDs-GSH. 
in the basal membrane and alveolar epithelium. ${ }^{28}$ These data correlate with previous studies, using QDs and fluorescence microscopy, indicating that 5 minutes after administration of B16-BL6 cells into the blood stream, these cells accumulated in small lung vessels. ${ }^{45}$

Several studies have shown that the majority of QDs present in the blood stream of mice rapidly accumulate in reticuloendothelial system organs (liver and spleen) and are excluded from heart and thymus. ${ }^{51-54}$ Nevertheless, no significant fluorescence increase in the spleen was detected when B16F10 ${ }_{\text {QDs-GSH-10NAC }}$ cells were injected (Figure 6I), while fluorescence accumulation in the thymus was detected between 30 minutes and 2 hours post-injection (Figure 6J). These results suggest that rQDs-GSH remain confined within $\mathrm{B} 16 \mathrm{~F} 10$ cells and are in agreement with a previous report by Voura et al, showing that QDs were not released from QDs-DHLA-labeled B16F10 cells. ${ }^{12}$

The thymus is a lymphatic, endocrine, and immune organ where T cells mature and are released to the circulation. ${ }^{53}$ The cytotoxic T-cells, as well as natural killer T-cells, specifically participate in the early clearance of tumor cells from the blood stream. ${ }^{54,55}$ Given that the blood-thymus barrier prevents direct contact between the thymus and components of the blood stream, ${ }^{56,57}$ the observed pronounced accumulation of fluorescence in the thymus suggests that B $16 \mathrm{~F} 10_{\text {QDs-GSH-10NAC }}$ are being rapidly recognized by $\mathrm{T}$ cells.

Our results indicated that B $16 \mathrm{~F} 10_{\mathrm{QDs}-\mathrm{GSH}-10 \mathrm{NAC}}$ cells were rapidly distributed ( 5 minutes) to the heart, liver, kidneys, and lungs of C57BL/6 mice and likely accumulated there by an embolic arrest process. ${ }^{24}$ However, despite the initial generic distribution, fluorescence intensity decreases in the other organs and a selective enrichment of B16F10 ${ }_{\text {QDs-GSH-10NAC }}$ cells in lungs is observed within the first few hours post-injection. This suggests that $\mathrm{B} 16 \mathrm{~F} 10_{\mathrm{QDs}-\mathrm{GSH}-10 \mathrm{NAC}}$ cells maintain the "homing" reported previously, ${ }^{24,25,45}$ and thus have the potential to be used as a tool for assessing early accumulation/ distribution of metastatic cells.

To confirm the presence of $\mathrm{B} 16 \mathrm{~F} 10_{\mathrm{QDs}-\mathrm{GSH}-10 \mathrm{NAC}}$ cells in the lungs of $\mathrm{C} 57 \mathrm{BL} / 6$ mice, lung sections obtained 6 hours post-injection of cells into the tail vein of $\mathrm{C} 57 \mathrm{BL} / 6$ mice were analyzed by immunohistochemistry (Figure 7). Fluorescence due to $\mathrm{B} 16 \mathrm{~F} 10_{\mathrm{QDS}-\mathrm{GSH}-10 \mathrm{NAC}}$ presence was observed in histological slices from mice and was clearly distinguishable from the background fluorescence of controls (Figure 7A), confirming the presence of B16F10 ${ }_{\text {QDs-GSH-10NAC }}$ cells in the lungs of C57BL/6 mice. During colonization of distant organs, the extravasation process is crucial. ${ }^{58}$ Given that $\mathrm{B} 16 \mathrm{~F} 10$ cells specifically target the lung in metastasis assays, it was important to determine whether B16F10 ${ }_{\text {QDs-GSH-10NAC }}$ cells were capable of transmigrating through the vascular endothelium and colonizing the lungs in vivo. With this in mind, B16F10 ${ }_{\text {QDs-GSH-10NAC }}$ and Calcein AM-labeled B16F10 $\left(\mathrm{B} 16 \mathrm{~F} 10_{\text {Calcein }}\right)$ cells were compared by histological analysis of lung sections 6 and 24 hours post-injection of cells into the tail vein of C57BL/6 mice. Specifically, we determined the fluorescence associated with B16F10 ${ }_{\mathrm{QDs}-\mathrm{GSH}-10 \mathrm{NAC}}$ and B16F10 ${ }_{\text {Calcein }}$ cells in the endothelial vasculature, alveolae, and bronchi (Figure S5A and B). B16F10 ${ }_{\text {QDs-GSH-10NAC }}$ and $\mathrm{B} 16 \mathrm{~F} 10_{\text {Calcein }}$ cells were mostly found in the perialveolar area, after 6 and 24 hours (Figure 7B and C). In addition, the number of cells present in those areas increased after 24 hours (Figure 7C), suggesting that the B16F10 ${ }_{\text {QDs-GSH-10NAC }}$ cells transmigrated to the same extent as B $16 \mathrm{~F} 10_{\text {Calcein }}$ cells. These results are consistent with previously published studies indicating that QDs-DHLA-labeled B16F10 cells transmigrate in a manner similar to non-labeled B16F10 cells. However, the mechanism by which transmigration of QDs-labeled cells occurs had not been elucidated yet.

In this context, it is worth noting that transmigration can occur by at least three different processes or combinations of thereof: 1) mesenchymal cell invasion (protease dependent), 2) collective cell invasion (protease dependent), and 3) amoeboid cell invasion (protease independent). ${ }^{58}$ Melanoma cells can invade using all three mechanisms and/or may switch from protease-dependent to amoeboid cell invasion. ${ }^{58,59}$ Amoeboid invasion may explain why B16F10 ${ }_{\text {QDs-GSH-10NAC }}$ cells, that are non-invasive in a protease-dependent invasion assay (Figure 3C and E), display the same in vivo transmigration ability as B16F10 ${ }_{\text {Calcein }}$ control cells (Figure 7B and $\mathrm{C}$ ).

As shown in Figure S6, B16F10 $0_{\text {QDs-GSH-10NAC }}$ cells (Figure S6A, Quadrant LR) and B16F10 ${ }_{\text {Calcein }}$ cells (Figure S6B, Quadrant UL) exhibit similar MFI. Therefore, a similar intensity would be expected when these cells are present in organs. A decrease in fluorescence intensity was observed between 6 and 24 hours for rQDs-GSH and Calcein-labeled cells (Figure S6C). This result could explain the decrease in fluorescence observed in lungs after 24 hours (Figure 6E) and indicates that B16F10 ${ }_{\text {QDs-GSH-10NAC }}$ cells are still present in the lungs at this time but with decreased fluorescence intensity. Also, these results indicate that 6 hours post-injection represents the optimal time point for the tracking of organ-specific B16F10 cell accumulation and permits avoiding the possible loss of signal due to decreases in B16F10 cell fluorescence during the tracking process.

Of note, the fluorescence intensity due to rQDs-GSH was significantly higher than that associated with Calcein after 24 hours (Figure S6C), suggesting that the rQDs-GSH labeling is more stable over time than fluorescence labeling using Calcein. 
A
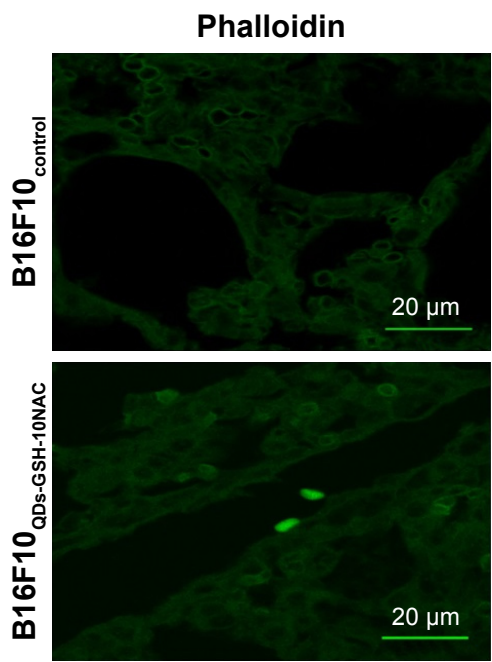

B
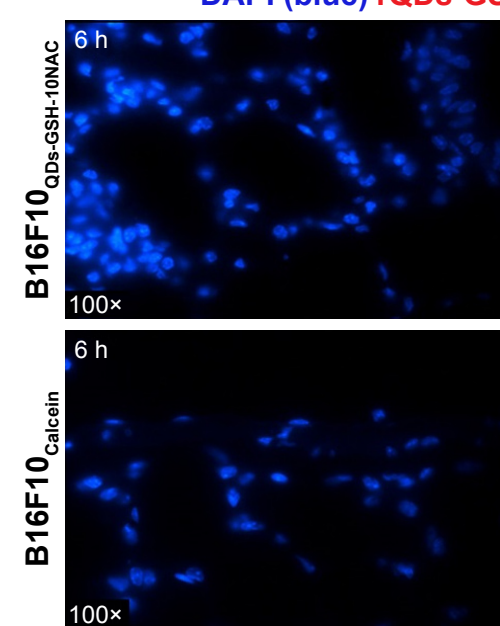

C
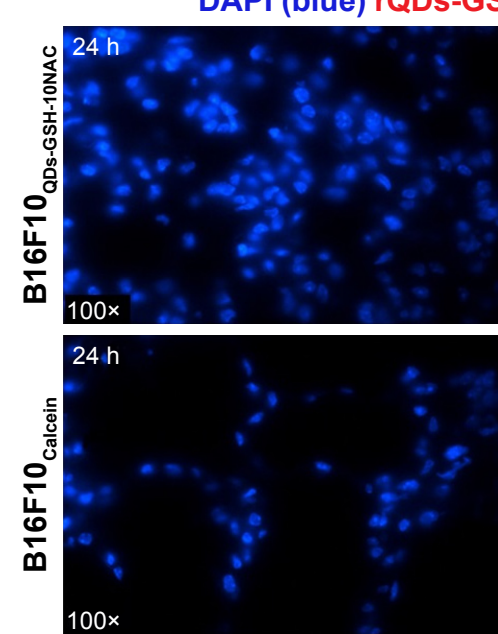

rQDs-GSH
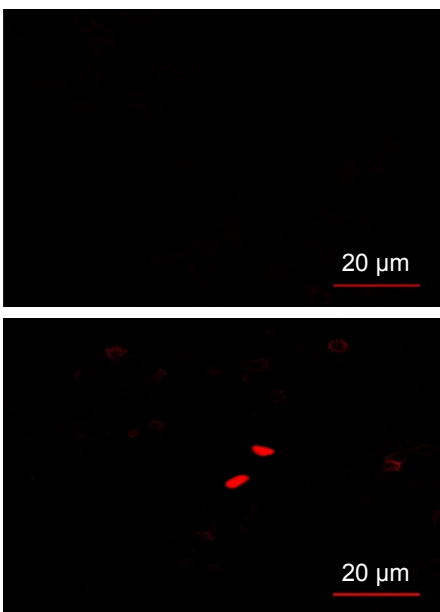

$\mu \mathrm{m}$
Merge
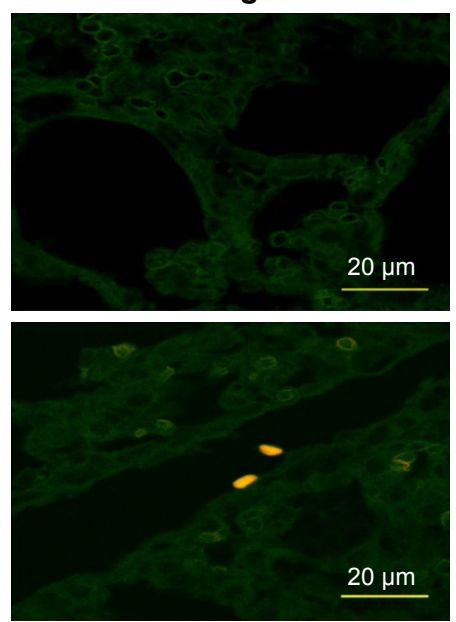

Merge
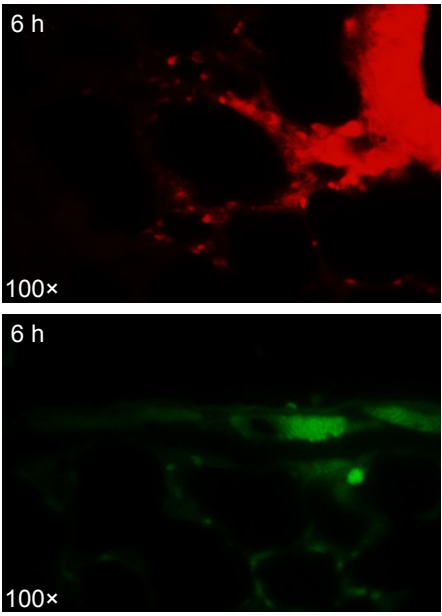

$100 \times$
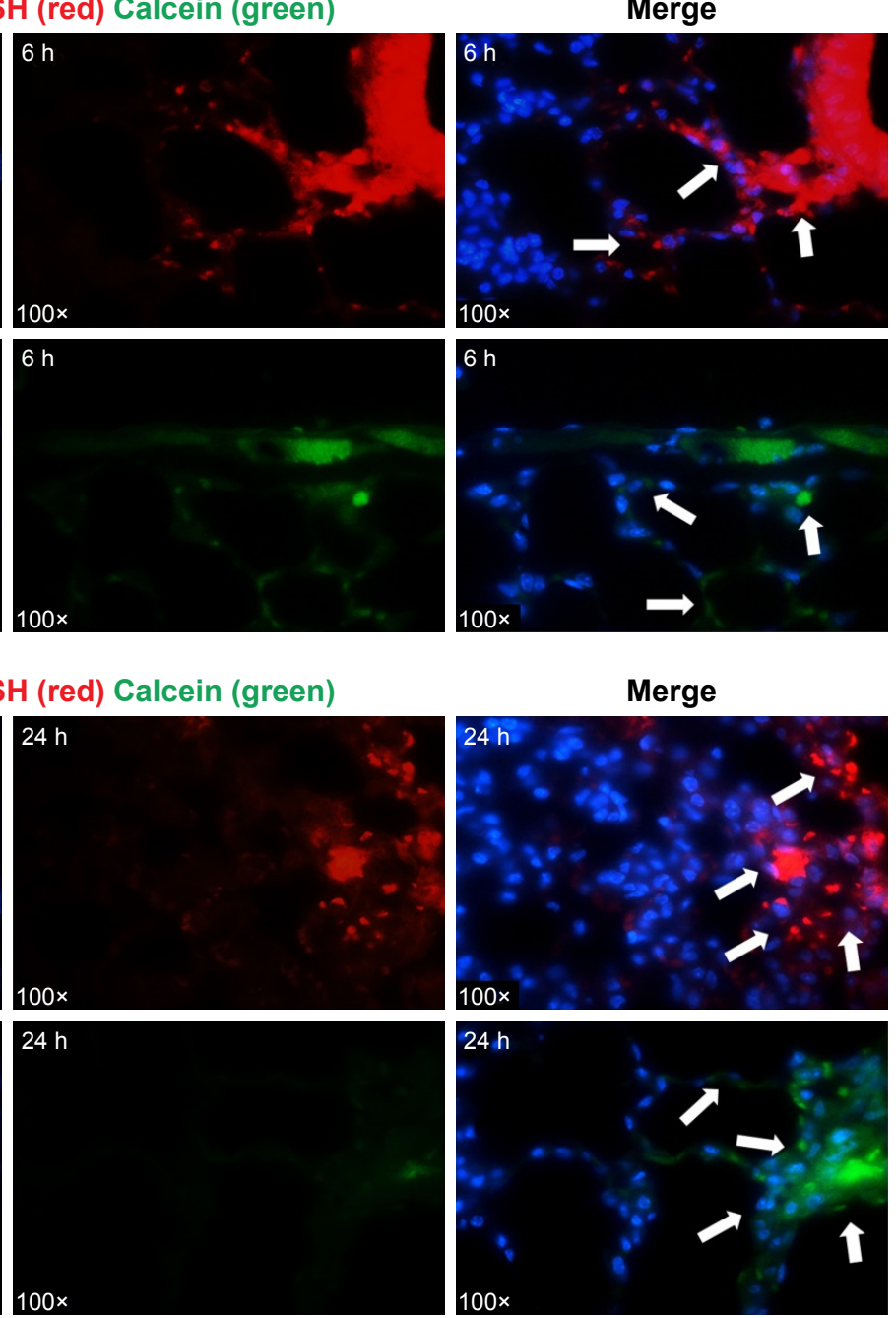

Figure 7 Presence and distribution of $\mathrm{BI} 6 \mathrm{FIO}{ }_{\mathrm{QDs}-\mathrm{GSH}-10 \mathrm{NAC}}$ and $\mathrm{BI} 6 \mathrm{FIO}{ }_{\text {Calcein }}$ cells in the lungs of $\mathrm{C} 57 \mathrm{BL} / 6$ mice.

Notes: $\mathrm{BI}_{6 \mathrm{FIO}} \mathrm{QDs}-\mathrm{GSH}-10 \mathrm{NAC}, \mathrm{BI6FI0}{ }_{\text {Calcein }}$, and control cells were injected into the tail vein of C57BL/6 mice. Fluorescence signals due to either rQDs-GSH or Calcein were determined in lungs at 6 and 24 hours post-injection. (A) Confocal images of histological sections from lungs collected at 6 hours post-injection of BI6FI0 ${ }_{\text {QDs-GSH-IONAC }}$ or $\mathrm{BI}_{6 \mathrm{FIO}}$ cells. (B) Confocal images of histological sections from lungs collected at 6 hours post-injection of BI6FI0 ${ }_{\text {ODs-GSH-IONAC }}$ or BI6FI0 of histological sections from lungs collected at 24 hours post-injection of $\mathrm{BI} 6 \mathrm{FIO}{ }_{\mathrm{QDS}-\mathrm{GSH}-10 \mathrm{NAC}}$ or BI6FI0 Calcein cells. White arrows show fluorescence due to presence of $\mathrm{BI} 6 \mathrm{FIO}_{\mathrm{QDS}-G S H-I O N A C}$ or BI6FIO ${ }_{\text {Calcein }}$ cells. Note that the data shown were obtained in a double-blind study.

Abbreviations: $\mathrm{BI}_{6 \mathrm{FIO}} \mathrm{QDS}-\mathrm{GSH}-\mathrm{IONAC} \mathrm{BI6FI0}$ cells labeled with rQDs-GSH in presence of $10 \mathrm{mM}$ of NAC; GSH, glutathione; NAC, N-acetylcysteine; QDs, quantum dots; rQDs-GSH, red QDs-GSH. 
Finally, these results demonstrate that our methodology of QDs-GSH-labeling cells allows obtaining nearly $100 \%$ viable labeled cells with increased fluorescence signal. In addition, these cells can migrate, transmigrate, and maintain their "homing," similar to the unlabeled B16F10 cells. Thus, B16F10 ${ }_{\text {QDs-GSH-10NAC }}$ cells can be used to track the early distribution/accumulation of B16F10 cells in C57BL/6 organs by in vivo fluorescence imaging, without the need for fluorescence microscopy and, in doing so, monitor early steps involved in the colonization of distant organs.

\section{Proof of concept}

According to Sahai 2007, "the development of imaging studies can be important for preclinical evaluation of drugs that target steps in the metastatic process." ${ }^{\prime 4}$ Our results showed that B16F10 ${ }_{\text {QDs-GSH-10NAC }}$ cells can be used to track in vivo migration and the accumulation/distribution of metastatic cells in C57BL/6 mice independently of the invasion process. We believe that this model has the potential to be used as a tool to determine more specifically how expression of different proteins in these cells and/or the application of drugs affect migration, accumulation, and/or distribution of B16F10 ${ }_{\text {QDs-GSH-10NAC }}$ cells in C57BL/6 mice.

CAV-1 is an integral membrane protein, which has been shown to increase the migration, invasion, and metastasis of B16F10 cells. ${ }^{25,60}$ With this in mind, we performed a proofof-concept experiment, using B16F10 cells stably transfected with either the empty pLacIOP vector [B16F10(Mock)

A

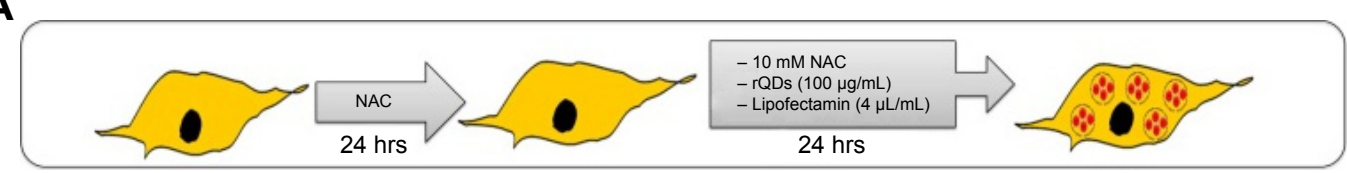

B

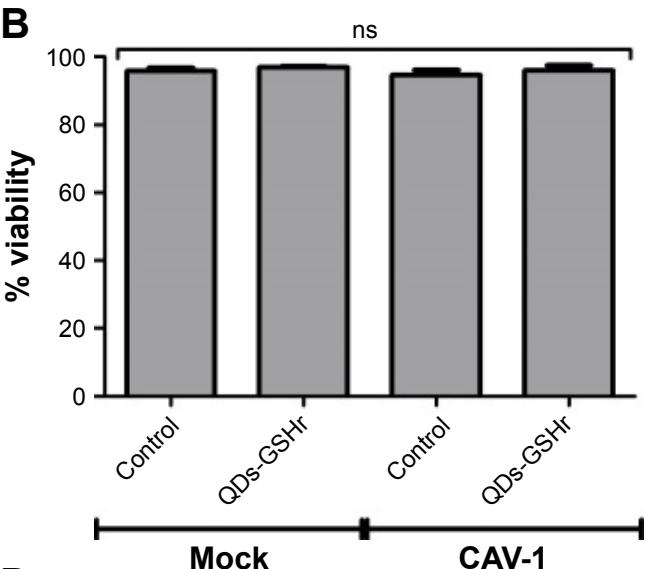

D

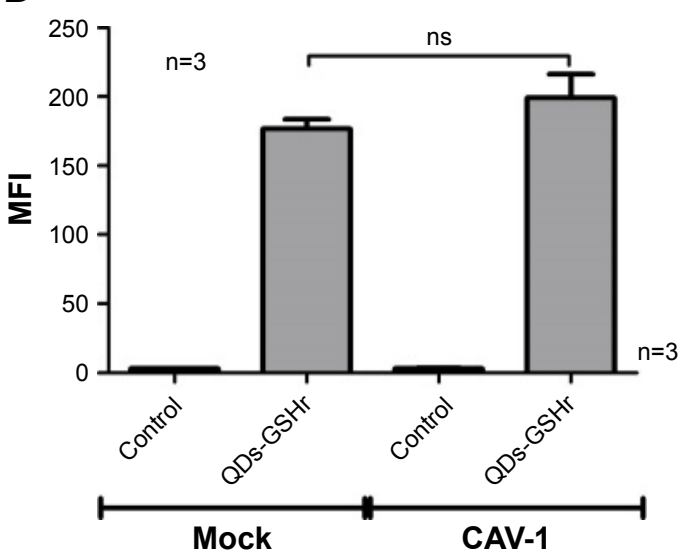

C

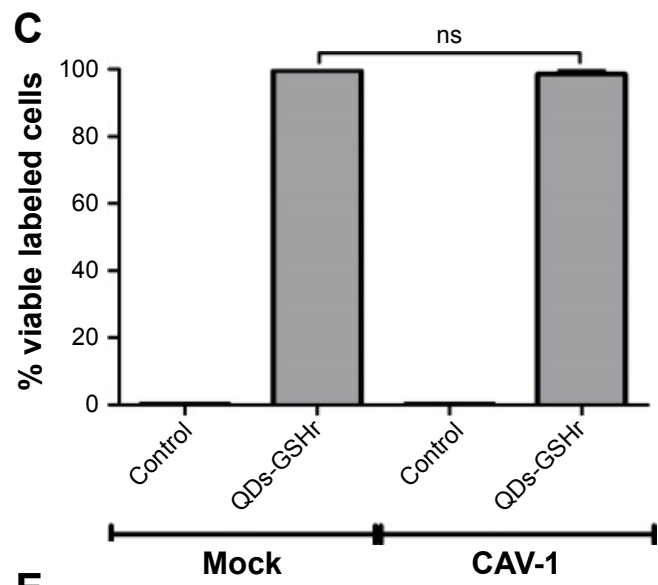

E

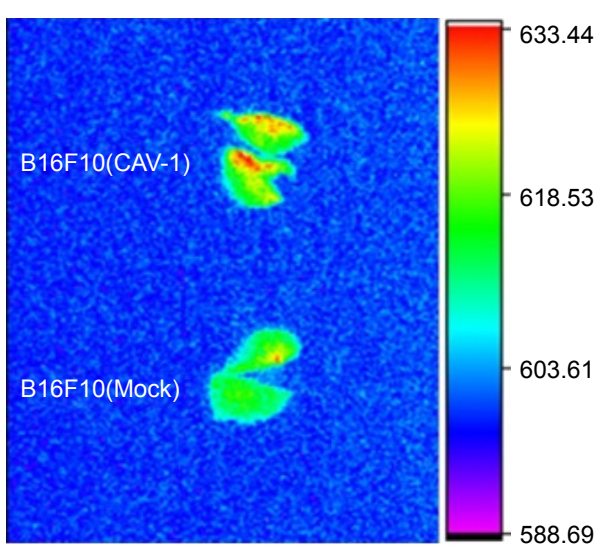

Figure 8 Proof-of-concept experiment to evaluate the effect of CAVI expression in labeled BI6FI0 cells following injection into C57BL/6 mice.

Notes: BI6FIO(Mock) and BI6FI0(CAV-I) cells were labeled with rQDs-GSH and used in this proof-of-concept experiment. (A) Schematic summarizing the labeling assays of BI6FI0(Mock) or BI6FI0(CAV-I) cells used in proof of concept. (B) Total viability of BI6FIO(Mock) and BI6FI0(CAV-I) cells after labeling with rQDs-GSH. (C) Viability rQDs-GSH-labeled BI6FIO(Mock) and BI6FIO(CAV-I) cells. (D) MFI of viable rQDs-GSH-labeled BI6FIO(Mock) and BI6FIO(CAV-I) cells. (E) In vivo imaging of lungs collected from C57BL/6 mice 6 hours post-injection with viable rQDs-GSH-labeled BI6FIO(Mock) and BI6FI0(CAV-I) cells. Results were averaged from three independent experiments $(n=3)$. Data were analyzed using the nonparametric Mann-Whitney test. Treated cells that did not show statistically significant differences are indicated. Note that the data shown were obtained in a double-blind study.

Abbreviations: CAV-I, Caveolin-I; GSH, glutathione; MFI, mean fluorescence intensity; QDs, quantum dots; rQDs-GSH, red QDs-GSH. 
cells] or the vector containing the CAV1-encoding insert [B16F10(CAV-1) cells] that were then labeled with rQDsGSH as described in Figure 8A (B16F10 ${ }_{\text {QDs-GSH-10NAC }}$ ). For the sake of simplicity, such labeled cells are referred to here as B16F10(Mock) and B16F10(CAV1) cells. Following labeling, we found that overall viability (Figure 8B), as well as the viability of labeled cells (Figure 8C), as determined by flow cytometry, were similar for B16F10(Mock) and B16F10(CAV1) cells. Moreover, the MFI was also similar (Figure 8D) for both types of cells as determined by flow cytometry. These results indicate that overexpression of CAV-1 in B16F10 cells neither altered the labeling with rQDs-GSH nor the viability of labeled B16F10 cells. B16F10 cells do not express PTRF/Cavin-1,28,61 and also lack the presence of caveolae-like structures at the cell surface. Moreover, immunofluorescence studies have shown that the large majority of the CAV1 protein in B16F10(CAV1) cells is intracellular, predominantly in a perinuclear location, ${ }^{28}$ consistent with the notion that the presence of CAV1 in B16F10(CAV1) cells does not modulate QDs-GSH internalization.

Subsequently, $2 \times 10^{5}$ viable and rQDs-GSH-labeled B16F10(Mock) or B16F10(CAV-1) cells were injected into the tail vein of $\mathrm{C} 57 \mathrm{BL} / 6$ mice, and 6 hours post-injection lungs from those recipient mice were collected for ex vivo fluorescence tracking using IVIS. As shown in Figure 8E, fluorescence intensity observed in lungs was greater for mice injected with B16F10(CAV-1) cells than those that received B16F10(Mock) cells. This result suggests that enhanced expression of CAV-1 in B16F10 melanoma cells promotes experimental lung metastasis described previously by our group by enhancing the accumulation of B16F10 cells in the lungs of recipient mice. In doing so, these experiments validate the potential of using rQDs-GSH-labeled cells to monitor early steps in metastasis.

\section{Conclusion}

In the present study, we describe for the first time the effects of biomimetic QDs-GSH incorporation on the viability and metastatic behavior of B16F10 cells. Also, we define a novel methodology that permits ameliorating detrimental consequences of QDs-GSH uptake. QDs-GSH' incorporation augments ROS and cell migration in B16F10 cells; however, these effects can be corrected by NAC treatment. In addition, NAC treatment allows increasing significantly the viability and fluorescence intensity of rQDs-GSH-labeled cells. However, proliferation and invasiveness of B16F10 cells, as well as the formation of metastatic lung nodules, were all attenuated by QDs-GSH internalization, probably as a consequence of cadmium present in the QDs-GSH core.
Moreover, we show that QDs-GSH-labeled B16F10 cells can be used to track the in vivo migration and early distribution/ accumulation of these cells in C57BL/6 organs. Our study reveals that these cells preferentially accumulated in lungs of C57BL/ 6 mice as early as 5 minutes post-injection. Thus, although QDs-GSH internalization blocks the ability of B16F10 cells to form nodules in the lung, QDs-GSH-labeled B16F10 cells can be successfully tracked during the early steps involved in the colonization of distant organs.

Finally, our study provides a cell-labeling methodology that should be useful for characterizing the early steps of tissue-specific accumulation and distribution of B16F10 cells in C57BL/6 mice, as well as help to evaluate the effects of drugs and/or different biological factors (cytokines, signaling proteins, etc.) on these processes and, in doing so, improve our understanding of these events. With this in mind, we propose that QDs-GSH can be expected to have considerable potential for future use in cancer theranostics.

\section{Acknowledgments}

The authors appreciate the support of Cellular Communication Laboratory, Faculty of Medicine, Universidad de Chile; The BioNanotechnology and Microbiology Laboratory, Faculty of Biological Sciences, Universidad Andres Bello; The Department of Pharmacological and Toxicological Chemistry, Faculty of Chemical and Pharmaceutical Sciences, Universidad de Chile and Fundación Ciencia y Vida, Santiago, Chile. The authors also appreciate the personal support of the Díaz-García family (VMDG) and Erika Elcira Donoso Lopez (JMPD). The study was funded by CONICYT-FONDAP 15130011 (AQ), FONDECYT 1130250, 1170925 (AQ), Anillo ACT 1111 (AQ, JMPD), FONDECYT 1151255 (JMPD), INACH RT-25-16 (JMPD), AFOSR FA9550-15-1-0140 (JMPD), and Basal de FCV PFB-16 (LLG). A post-doctoral CONICYT award 3140463 (SG) and a CONICYT PhD fellowship 21120816 (VMDG) are also acknowledged.

\section{Author contributions}

All authors contributed toward data analysis, drafting and critically revising the paper and agree to be accountable for all aspects of the work.

\section{Disclosure}

The authors report no conflicts of interest in this work.

\section{References}

1. Ferlay J, Shin HR, Bray F, Forman D, Mathers C, Parkin DM. Estimates of worldwide burden of cancer in 2008: GLOBOCAN 2008. Int J Cancer. 2010;127(12):2893-2917. 
2. Chaffer CL, Weinberg RA. A perspective on cancer cell metastasis. Science. 2011;331(6024):1559-1564.

3. Valastyan S, Weinberg RA. Tumor metastasis: molecular insights and evolving paradigms. Cell. 2011;147(2):275-292.

4. Sahai E. Illuminating the metastatic process. Nat Rev Cancer. 2007; 7(10):737-749.

5. Chinen AB, Guan CM, Ferrer JR, Barnaby SN, Merkel TJ, Mirkin CA. Nanoparticle Probes for the Detection of Cancer Biomarkers, Cells, and Tissues by Fluorescence. Chem Rev. 2015;115(19):10530-10574

6. Fang M, Peng CW, Pang DW, Li Y. Quantum dots for cancer research: current status, remaining issues, and future perspectives. Cancer Biol Med. 2012;9(3):151-163.

7. Chen C, Peng J, Sun SR, Peng CW, Li Y, Pang DW. Tapping the potential of quantum dots for personalized oncology: current status and future perspectives. Nanomedicine. 2012;7(3):411-428.

8. Pérez-Donoso JM, Monrás JP, Bravo D, et al. Biomimetic, mild chemical synthesis of CdTe-GSH quantum dots with improved biocompatibility. PLoS One. 2012;7(1):e30741.

9. Díaz V, Ramírez-Maureira M, Monrás JP, et al. Spectroscopic properties and biocompatibility studies of CdTe Quantum Dots capped with biological thiols. Sci Adv Mater. 2012;4(5):609-616.

10. Zheng Y, Gao S, Ying JY. Synthesis and Cell-Imaging Applications of Glutathione-Capped CdTe Quantum Dots. Adv Mater. 2007;19(3):376-380.

11. Rosenblum LT, Kosaka N, Mitsunaga M, Choyke PL, Kobayashi H. In vivo molecular imaging using nanomaterials: general in vivo characteristics of nano-sized reagents and applications for cancer diagnosis. Mol Membr Biol. 2010;27(7):274-285.

12. Voura EB, Jaiswal JK, Mattoussi H, Simon SM. Tracking metastatic tumor cell extravasation with quantum dot nanocrystals and fluorescence emission-scanning microscopy. Nat Med. 2004;10(9):993-998.

13. Derfus AM, Chan WCW, Bhatia SN. Probing the Cytotoxicity Of Semiconductor Quantum Dots. Nano Lett. 2004;4(1):11-18.

14. Hardman R. A toxicologic review of quantum dots: toxicity depends on physicochemical and environmental factors. Environ Health Perspect. 2006;114(2):165-172.

15. Rzigalinski BA, Strobl JS. Cadmium-containing nanoparticles: perspectives on pharmacology and toxicology of quantum dots. Toxicol Appl Pharmacol. 2009;238(3):280-288.

16. Ochi T, Otsuka F, Takahashi K, Ohsawa M. Glutathione and metallothioneins as cellular defense against cadmium toxicity in cultured Chinese hamster cells. Chem Biol Interact. 1988;65(1):1-14.

17. Anderson ME. Glutathione: an overview of biosynthesis and modulation. Chem Biol Interact. 1998;111-112:1-14.

18. Valko M, Morris H, Cronin MT. Metals, toxicity and oxidative stress. Curr Med Chem. 2005;12(10):1161-1208.

19. Peng L, He M, Chen B, Qiao Y, Hu B. Metallomics Study of CdSe/ZnS Quantum Dots in HepG2 Cells. ACS Nano. 2015;9(10):10324-10334.

20. Loo G. Redox-sensitive mechanisms of phytochemical-mediated inhibition of cancer cell proliferation (review). J Nutr Biochem. 2003; 14(2):64-73.

21. Xu G, Zeng S, Zhang B, Swihart MT, Yong KT, Prasad PN. New Generation Cadmium-Free Quantum Dots for Biophotonics and Nanomedicine. Chem Rev. 2016;116(19):12234-12327.

22. Pérez-Donoso JM, Monrás JP. Osorio-Roman IO, Vásquez CC; Universidad de Santiago de Chile. Synthesis of Highly Fluorescent GSH-CdTe Nanoparticles (Quantum Dots). United States patent US20130284979. 2017 Aug 15.

23. Gautier JL, Monrás JP, Osorio-Román IO, et al. Surface characterization of GSH-CdTe quantum dots. Mater Chem Phys. 2013;140(1): 113-118.

24. Fidler IJ. Biological behavior of malignant melanoma cells correlated to their survival in vivo. Cancer Res. 1975;35(1):218-224.

25. Lobos-González L, Aguilar L, Diaz J, et al. E-cadherin determines Caveolin-1 tumor suppression or metastasis enhancing function in melanoma cells. Pigment Cell Melanoma Res. 2013;26(4):555-570.

26. Ellman GL. Tissue sulfhydryl groups. Arch Biochem Biophys. 1959; 82(1):70-77.
27. Riener CK, Kada G, Gruber HJ. Quick measurement of protein sulfhydryls with Ellman's reagent and with 4,4'-dithiodipyridine. Anal Bioanal Chem. 2002;373(4-5):266-276.

28. Ortiz R, Díaz J, Díaz N, et al. Extracellular matrix-specific Caveolin-1 phosphorylation on tyrosine 14 is linked to augmented melanoma metastasis but not tumorigenesis. Oncotarget. 2016;7(26):40571-40593.

29. Yildirimer L, Thanh NT, Loizidou M, Seifalian AM. Toxicology and clinical potential of nanoparticles. Nano Today. 2011;6(6):585-607.

30. Lovrić J, Bazzi HS, Cuie Y, Fortin GR, Winnik FM, Maysinger D. Differences in subcellular distribution and toxicity of green and red emitting CdTe quantum dots. J Mol Med. 2005;83(5):377-385.

31. Schaeublin NM, Braydich-Stolle LK, Schrand AM, et al. Surface charge of gold nanoparticles mediates mechanism of toxicity. Nanoscale. 2011;3(2):410-420.

32. Panté N, Kann M. Nuclear pore complex is able to transport macromolecules with diameters of about $39 \mathrm{~nm}$. Mol Biol Cell. 2002;13(2): 425-434.

33. Catalan-Figueroa J, Palma-Florez S, Alvarez G, Fritz HF, Jara MO, Morales JO. Nanomedicine and nanotoxicology: the pros and cons for neurodegeneration and brain cancer. Nanomedicine (Lond). 2016;11(2): 171-187.

34. Wu WS. The signaling mechanism of ROS in tumor progression. Cancer Metastasis Rev. 2006;25(4):695-705.

35. Verma RP, Hansch C. Matrix metalloproteinases MMPs: chemical-biological functions and (Q)SARs. Bioorg Med Chem. 2007;15(6):2223-2268.

36. Kessenbrock K, Plaks V, Werb Z. Matrix metalloproteinases: regulators of the tumor microenvironment. Cell. 2010;141(1):52-67.

37. Khokha R, Zimmer MJ, Graham CH, Lala PK, Waterhouse P. Suppression of invasion by inducible expression of tissue inhibitor of metalloproteinase-1 (TIMP-1) in B16-F10 melanoma cells. $J$ Natl Cancer Inst. 1992;84(13):1017-1022.

38. Fröhlich E. Cellular elimination of nanoparticles. Environ Toxicol Pharmacol. 2016;46:90-94.

39. Jiang X, Röcker C, Hafner M, Brandholt S, Dörlich RM, Nienhaus GU. Endo- and exocytosis of zwitterionic quantum dot nanoparticles by live HeLa cells. ACS Nano. 2010;4(11):6787-6797.

40. Mahmoudi M, Azadmanesh K, Shokrgozar MA, Journeay WS, Laurent S. Effect of nanoparticles on the cell life cycle. Chem Rev. 2011;111(5):3407-3432.

41. Yang PM, Chiu SJ, Lin KA, Lin LY. Effect of cadmium on cell cycle progression in Chinese hamster ovary cells. Chem Biol Interact. 2004;149(2-3):125-136.

42. Bertin G, Averbeck D. Cadmium: cellular effects, modifications of biomolecules, modulation of DNA repair and genotoxic consequences (a review). Biochimie. 2006;88(11):1549-1559.

43. Kareem A, Zafar H, Sherwani A, Mohammad O, Khan TA. Synthesis, characterization and in vitro anticancer activity of 18 -membered octaazamacrocyclic complexes of $\mathrm{Co}(\mathrm{II}), \mathrm{Ni}(\mathrm{II}), \mathrm{Cd}(\mathrm{II})$ and $\mathrm{Sn}(\mathrm{II}) . J$ Mol Struct. 2014;1075:17-25.

44. Sreekanth TV, Pandurangan M, Dillip GR, Kim DH, Lee YR. Toxicity and efficacy of $\mathrm{CdO}$ nanostructures on the MDCK and Caki-2 cells. J Photochem Photobiol B. 2016;164:174-181.

45. Saitoh Y, Terada N, Ohno N, et al. Imaging of thrombosis and microcirculation in mouse lungs of initial melanoma metastasis with in vivo cryotechnique. Microvasc Res. 2014;91:73-83.

46. Wexler H. Accurate identification of experimental pulmonary metastases. J Natl Cancer Inst. 1966;36(4):641-645.

47. Choi HS, Liu W, Misra P, et al. Renal clearance of quantum dots. Nat Biotechnol. 2007;25(10):1165-1170.

48. Ye L, Yong KT, Liu L, et al. A pilot study in non-human primates shows no adverse response to intravenous injection of quantum dots. Nat Nanotechnol. 2012;7(7):453-458.

49. Schipper ML, Cheng Z, Lee SW, et al. MicroPET-based biodistribution of quantum dots in living mice. $J$ Nucl Med. 2007;48(9): 1511-1518.

50. Smith AM, Duan H, Mohs AM, Nie S. Bioconjugated quantum dots for in vivo molecular and cellular imaging. Adv Drug Deliv Rev. 2008; 60(11):1226-1240. 
51. Schipper ML, Iyer G, Koh AL, et al. Particle size, surface coating, and PEGylation influence the biodistribution of quantum dots in living mice. Small. 2009;5(1):126-134.

52. Haque MM, Im H-Y, Seo J-E, Hasan M, Woo K, Kwon O-S. Effects of folic acid and polyethylene glycol coated quantum dots on toxicity and tissue uptake to precision-cut spleen slices of rats. J Pharm Investig. 2013;43(5):375-383.

53. Miller JF. The discovery of thymus function and of thymus-derived lymphocytes. Immunol Rev. 2002;185(1):7-14.

54. Andersen MH, Pedersen LO, Capeller B, Bröcker EB, Becker JC, thor Straten P. Spontaneous cytotoxic T-cell responses against survivinderived MHC class I-restricted T-cell epitopes in situ as well as ex vivo in cancer patients. Cancer Res. 2001;61(16):5964-5968.

55. Whiteside TL, Herberman RB. The role of natural killer cells in immune surveillance of cancer. Curr Opin Immunol. 1995;7(5):704-710.

56. Kendall MD. Functional anatomy of the thymic microenvironment. J Anat. 1991;177(1):1-29.
57. Nishino M, Ashiku SK, Kocher ON, Thurer RL, Boiselle PM, Hatabu H. The thymus: a comprehensive review. Radiographics. 2006;26(2):335-348.

58. van Zij1 F, Krupitza G, Mikulits W. Initial steps of metastasis: cell invasion and endothelial transmigration. Mutat Res. 2011;728(1-2):23-34.

59. Hegerfeldt Y, Tusch M, Bröcker EB, Friedl P. Collective cell movement in primary melanoma explants: plasticity of cell-cell interaction, beta1-integrin function, and migration strategies. Cancer Res. 2002;62(7):2125-2130.

60. Diaz-Valdivia N, Bravo D, Huerta H, et al. Enhanced caveolin-1 expression increases migration, anchorage-independent growth and invasion of endometrial adenocarcinoma cells. BMC Cancer. 2015;15(1):463.

61. Hill MM, Daud NH, Aung CS, et al. Co-regulation of cell polarization and migration by caveolar proteins PTRF/Cavin-1 and caveolin-1. PLoS One. 2012;7(8):e43041. 


\section{Supplementary materials}
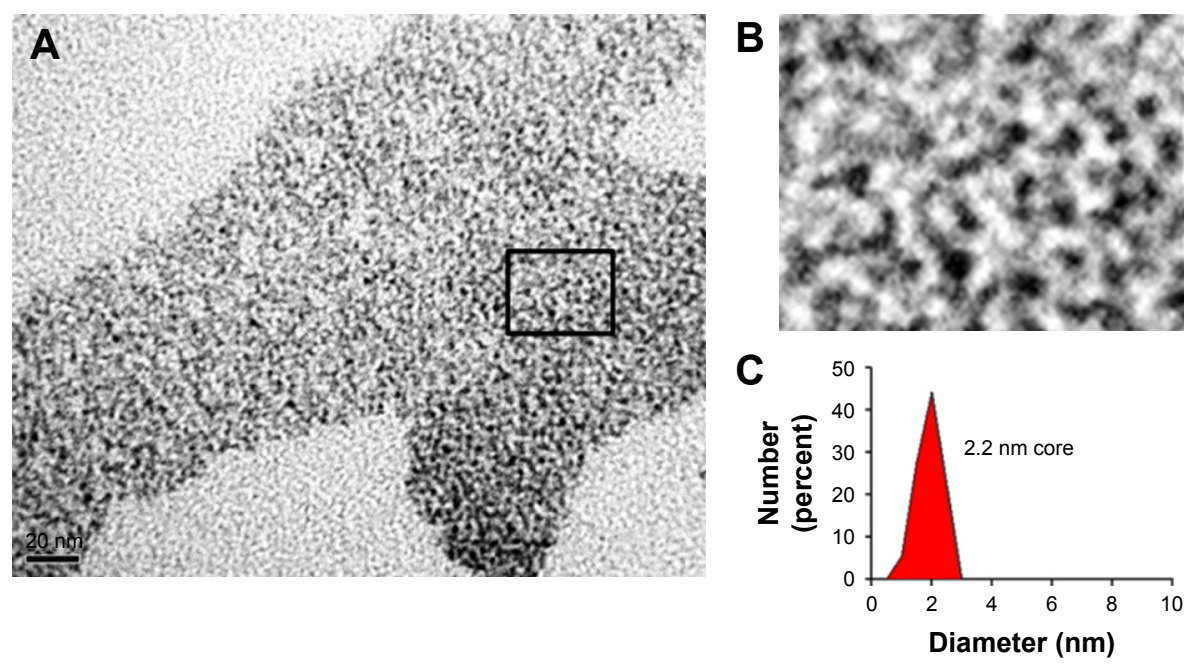

Figure SI TEM image of rQDs-GSH.

Notes: rQDs-GSH were observed by TEM microscopy and the core size was determined using Imagel software. (A) TEM image of rQDs-GSH. (B) A threefold amplification of square shown in image A. (C) Quantification of electron-dense granule size (core of rQDs-GSH).

Abbreviations: GSH, glutathione; QDs, quantum dots; rQDs-GSH, red QDs-GSH; TEM, transmission electron microscopy.

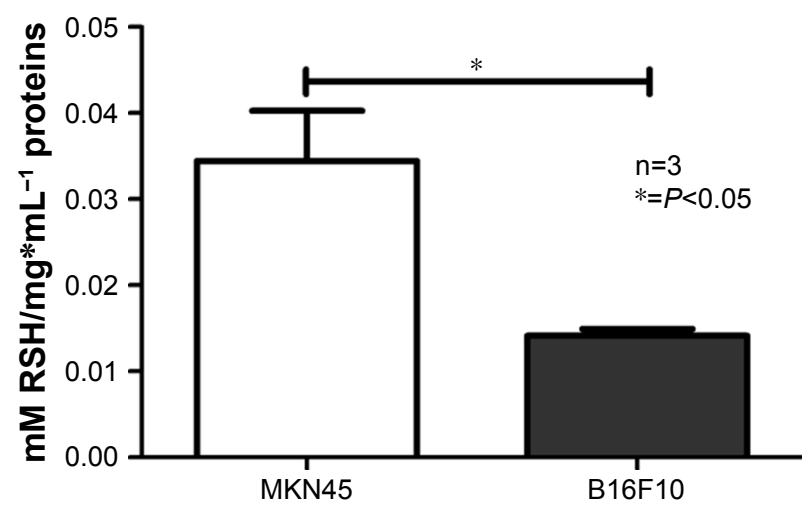

Figure S2 Intracellular reduced thiol levels in MKN45 and BI6FIO cells.

Notes: Intracellular RSH levels in MKN45 and BI6FIO cells were measured by Ellman's assay. Results were averaged from three independent experiments ( $\mathrm{n}=3$ ). Data were analyzed using the nonparametric Mann-Whitney test. Statistically significant differences are indicated.

Abbreviation: $\mathrm{RSH}$, reduced thiols. 
A

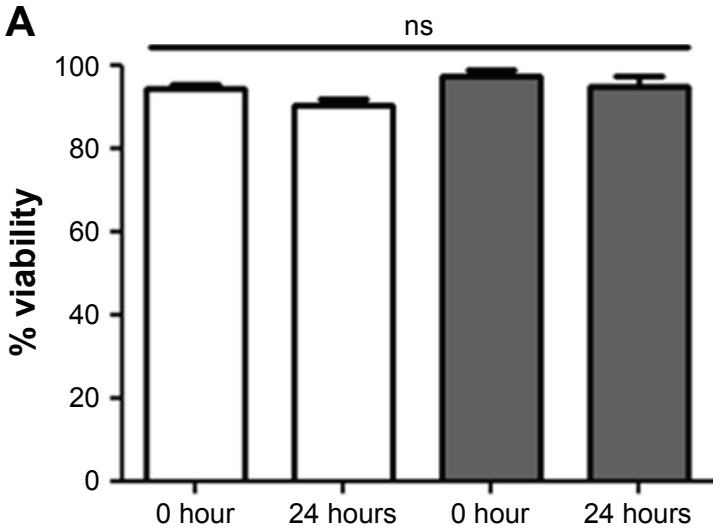

C

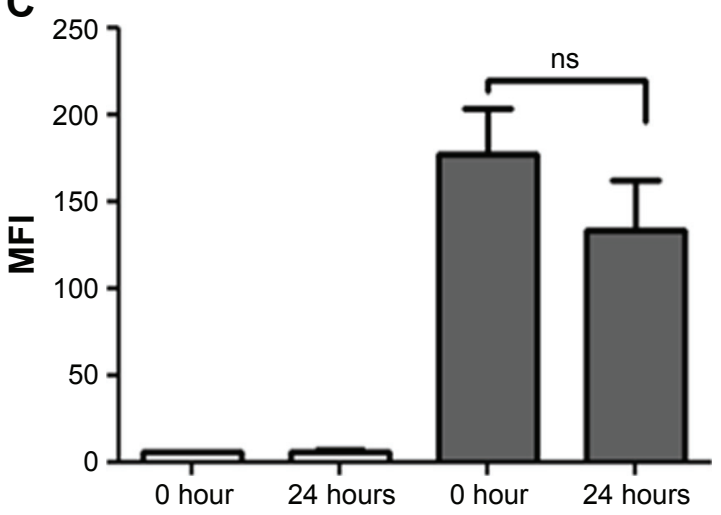

B

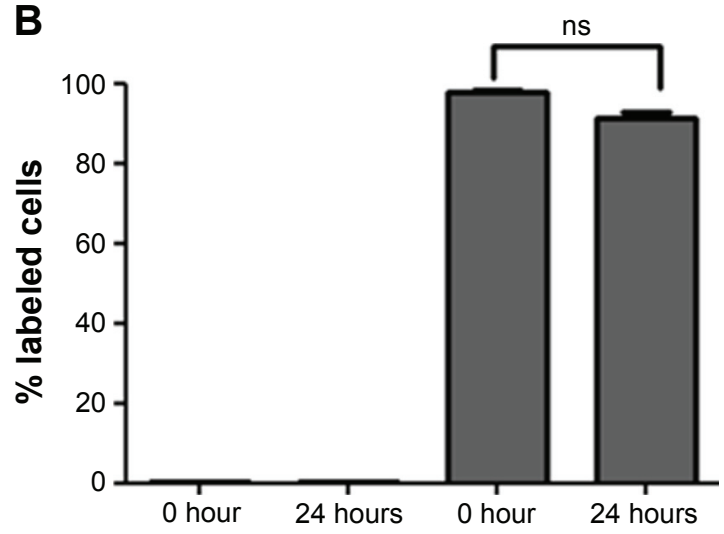

D

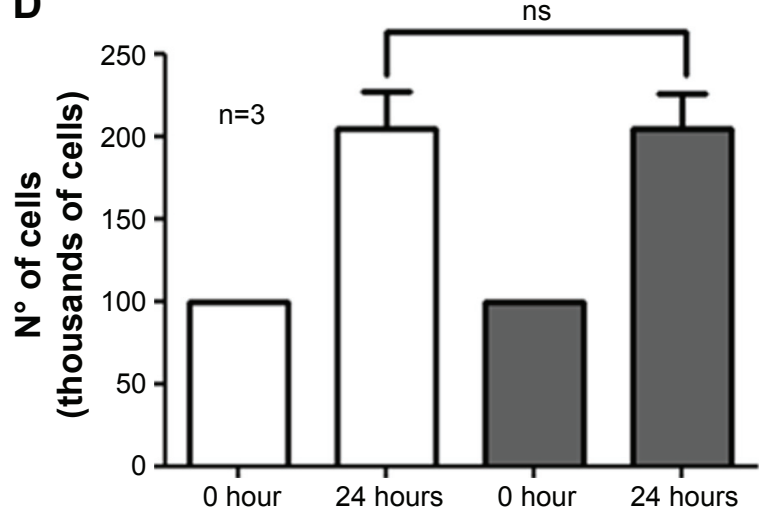

Figure S3 Effects of QDs-GSH incorporation on BI6FI0 cell proliferation and evaluation of QDs-GSH signal after 24 hours.

Notes: I $\times 10^{5} \mathrm{BI} 6 \mathrm{FIO}$ cells (white bars) and BI6FI0 cells labeled with rQDs-GSH (gray bars) were cultured in RPMI supplemented with $10 \%$ FBS for 24 hours, and cell viability, percentage of viable labeled cells, $\mathrm{MFI}$, and the total cell number (quantification of proliferation) were determined after cell labeling. (A) Cell viability at 0 or 24 hours post-labeling. (B) Percentage of viable BI6FI0 cells at 0 or 24 hours post-labeling. (C) MFI of viable BI6FI0 cells at 0 or 24 hours post-labeling. (D) Total number of BI6FI0 cells at 0 or 24 hours post-labeling. Results were averaged from three independent experiments $(n=3)$. Data were analyzed using the nonparametric Mann-Whitney test. The n.s. significant differences compared with the controls and different treatments are indicated.

Abbreviations: GSH, glutathione; MFI, mean fluorescence intensity; n.s., non-statistically; QDs, quantum dots; rQDs-GSH, red QDs-GSH.

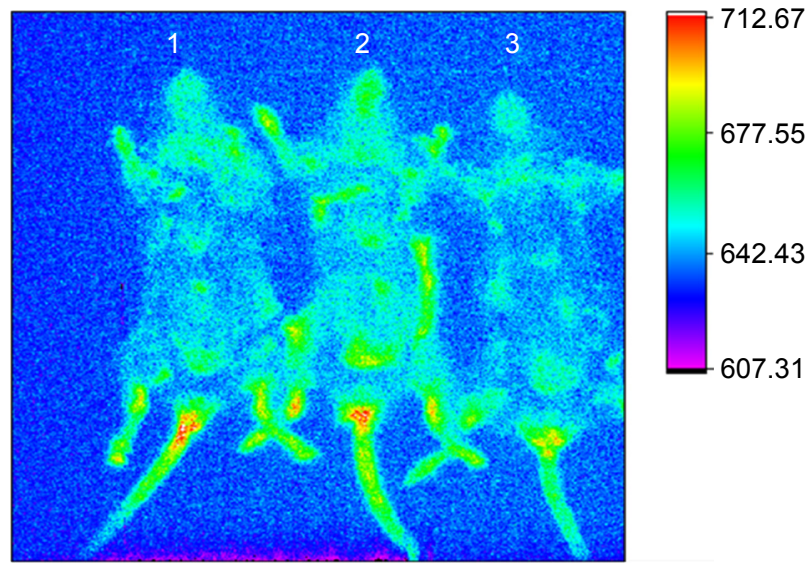

Figure S4 In vivo imaging of C57BL/6 mice treated with BI6FIO ${ }_{\mathrm{QDS}-\mathrm{GSH}-10 \mathrm{NAC}}$ and $\mathrm{BI} 6 \mathrm{FIO}$ control cells.

Notes: BI6FI0 ${ }_{\text {QDs-GSH-IONAC }}(\mathrm{I}$ and 3) and BI6FI0 control cells (2) were injected into C57BL/6 mice. Fluorescence signals for rQDs-GSH were followed in mice for 6 hours. Imaging shows no differences in fluorescence signals between the mice.

Abbreviations: $\mathrm{BI} 6 \mathrm{FIO}_{\mathrm{QDs}-\mathrm{GSH}-1 \mathrm{ONAC}}, \mathrm{BI} 6 \mathrm{FIO}$ cells labeled with rQDs-GSH in presence of $10 \mathrm{mM}$ of NAC; GSH, glutathione; MFI, mean fluorescence intensity; NAC, $\mathrm{N}$-acetylcysteine; QDs, quantum dots; rQDs-GSH, red QDs-GSH. 

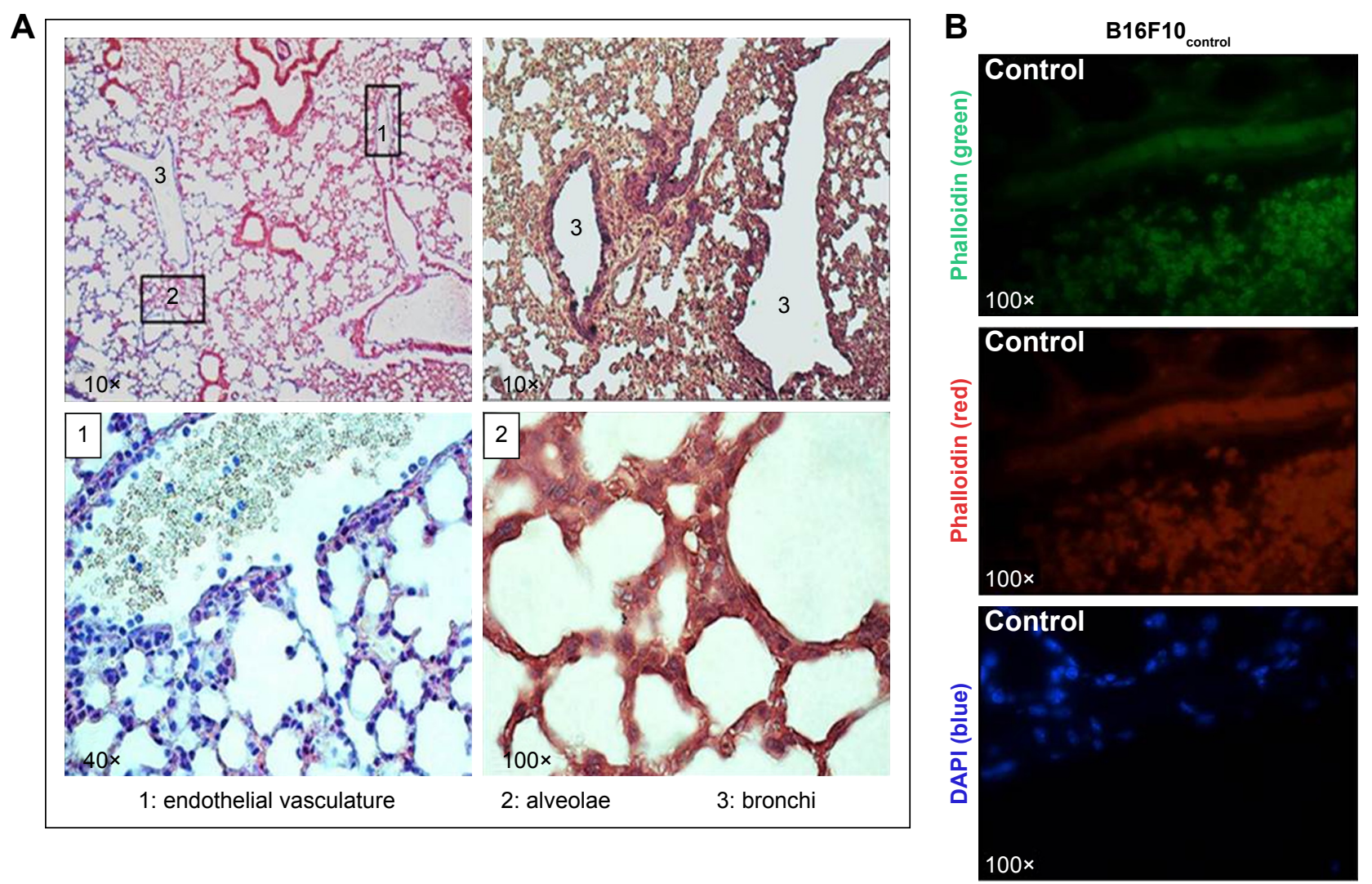

Figure S5 Controls of histological assays: fluorescence signals due to rQDs-GSH or Calcein were followed in lungs 6 hours post-injection of unlabeled BI6FI0 cells.

Notes: (A) Light microcopy images of histological sections from lungs collected 6 hours post-injection of unlabeled BI6FI0 cells and stained with hematoxylin and eosin. Images show various tissue areas where BI6FIO cells were identified. (B) Confocal images of histological sections from lungs collected 6 hours post-injection of unlabeled BI6FIO cells. Phalloidin green, red, and DAPI were used as a contrast media. No signals related to rQDs-GSH or Calcein were observed. Abbreviations: GSH, glutathione; QDs, quantum dots; rQDs-GSH, red QDs-GSH.

A

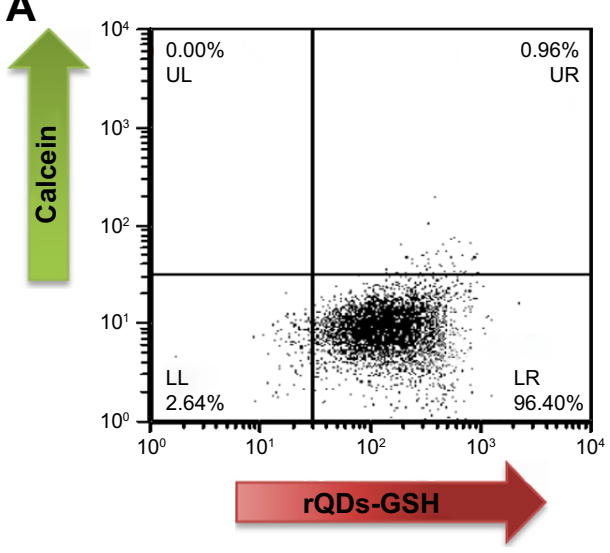

\begin{tabular}{|c|l|l|l|l|}
\hline Quadrant & $\begin{array}{l}\text { \# of } \\
\text { Events }\end{array}$ & $\begin{array}{l}\text { X } \\
\text { Geometric } \\
\text { mean }\end{array}$ & $\begin{array}{l}\text { Y } \\
\text { Geometric } \\
\text { mean }\end{array}$ & $\begin{array}{l}\text { \% of } \\
\text { Gated } \\
\text { Cells }\end{array}$ \\
\hline UL & 0 & 0.00 & 0.00 & 0.00 \\
\hline UR & 47 & 409.92 & 43.72 & 0.96 \\
\hline LL & 129 & 18.41 & 5.24 & 2.64 \\
\hline LR & 4,713 & 148.84 & 7.88 & 96.40 \\
\hline
\end{tabular}

B

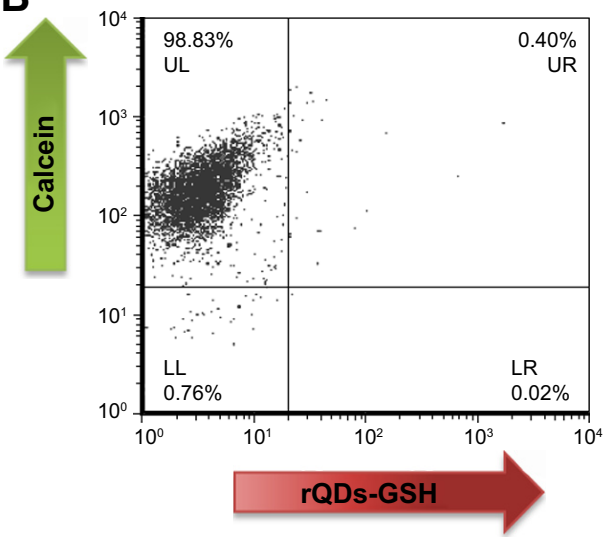

\begin{tabular}{|c|l|l|l|l|}
\hline Quadrant & $\begin{array}{l}\text { \# of } \\
\text { Events }\end{array}$ & $\begin{array}{l}\mathbf{X} \\
\text { Geometric } \\
\text { mean }\end{array}$ & $\begin{array}{l}\text { Y } \\
\text { Geometric } \\
\text { mean }\end{array}$ & $\begin{array}{l}\text { \% of } \\
\text { Gated } \\
\text { Cells }\end{array}$ \\
\hline UL & 5,227 & 3.05 & 167.46 & 98.83 \\
\hline UR & 21 & 50.59 & 380.18 & 0.40 \\
\hline LL & 40 & 3.34 & 6.55 & 0.76 \\
\hline LR & 1 & 21.48 & 15.40 & 0.02 \\
\hline
\end{tabular}

Figure S6 (Continued) 


\section{C}

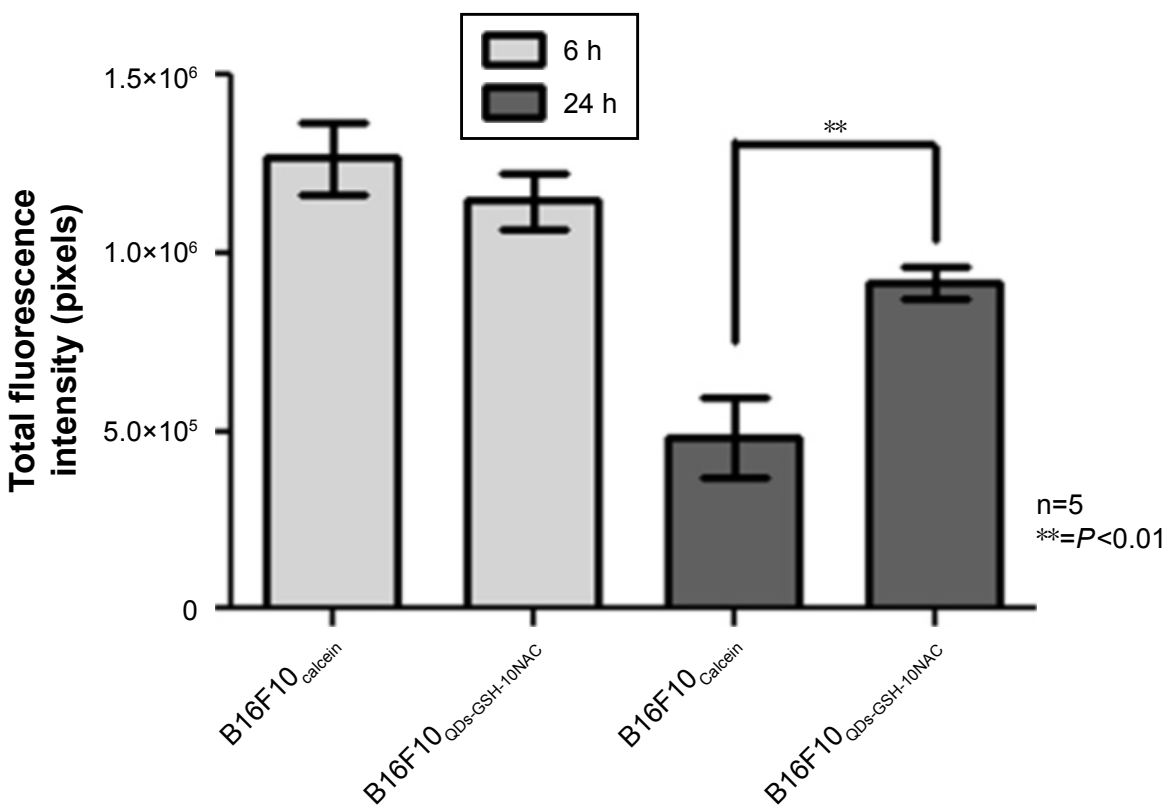

Figure S6 Fluorescence intensity of $\mathrm{BI} 6 \mathrm{FIO}{ }_{\mathrm{QDs}-G \mathrm{GH}-1 \mathrm{ONAC}}$ and $\mathrm{BI} 6 \mathrm{FIO}{ }_{\text {Calcein }}$ cells at 6 and 24 hours post-injection: dot plot obtained by flow cytometry and the respective quantification of mean fluorescence intensity in each quadrant.

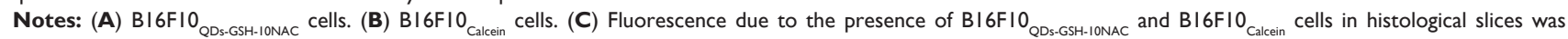
measured with ImageJ I.47 v software (National Institutes of Health, USA). Results were averaged from five independent experiments ( $\mathrm{n}=5$ ). Data were analyzed using the nonparametric Mann-Whitney test. Statistically significant differences are indicated.

Abbreviations: $\mathrm{BI6FI0}{ }_{\mathrm{QDs}-G S H-10 \mathrm{NAC}}, \mathrm{BI} 6 \mathrm{FIO}$ cells labeled with rQDs-GSH in presence of $10 \mathrm{mM}$ of NAC; GSH, glutathione; NAC, N-acetylcysteine; QDs, quantum dots; rQDs-GSH, red QDs-GSH.

\section{Publish your work in this journal}

The International Journal of Nanomedicine is an international, peerreviewed journal focusing on the application of nanotechnology in diagnostics, therapeutics, and drug delivery systems throughout the biomedical field. This journal is indexed on PubMed Central, MedLine, CAS, SciSearch $®$, Current Contents ${ }^{\circledR} /$ Clinical Medicine,
Journal Citation Reports/Science Edition, EMBase, Scopus and the Elsevier Bibliographic databases. The manuscript management system is completely online and includes a very quick and fair peer-review system, which is all easy to use. Visit http://www.dovepress.com/ testimonials.php to read real quotes from published authors. 